\title{
Spatial structure of neutron Cooper pair in low density uniform matter
}

\author{
Masayuki Matsuo \\ Department of Physics, Faculty of Science, Niigata University, Niigata 950-2181, Japan
}

(Dated: November 1, 2018)

\begin{abstract}
We analyze spatial structure of the neutron Cooper pair in superfluid low-density uniform matter by means of BCS calculations employing a bare force and the effective Gogny interaction. It is shown that the Cooper pair exhibits a strong spatial di-neutron correlation in a wide range of neutron density $\rho / \rho_{0} \approx 10^{-4}-0.5$. This feature is related to the crossover behavior between the pairing of the weak coupling BCS type and the Bose-Einstein condensation of bound neutron pairs. We also show that the zero-range delta interaction can describe the spatial structure of the neutron Cooper pair if the density dependent interaction strength and the cut-off energy are appropriately chosen. Parameterizations of the density-dependent delta interaction satisfying this condition are discussed.

PACS numbers: 21.60.Jz, 21.65.+f, 26.60.+c
\end{abstract}

\section{INTRODUCTION}

The importance of the pair correlation has been widely recognized for nucleon many-body systems in various circumstances, in particular in open-shell nuclei and in neutron stars. The pairing gap varies with system parameters such as $N, Z$ and the rotational frequency in the case of finite nuclei, or the temperature and the density in the case of neutron stars (cf. Refs. [1, 2, [3, [4, [5] as reviews). The pairing correlation at low nucleon density is of special interest since the theoretical predictions for low-density uniform matter suggest that the pairing gap may take, at around 1/10 of the normal nuclear density, a value which is considerably larger than that around the normal density 3, 4, 5. This feature is expected to have direct relevance to the properties of neutron stars, especially those associated with the inner crust [6, 7]. The strong pairing at low density may be relevant also to finite nuclei, if one considers neutron-rich nuclei near the drip-line [8, 9, 10, 11]. This is because such nuclei often accompany unsaturated low-density distribution of neutrons (the neutron skin and/or the neutron halo) surrounding the nuclear surface 12, 13, 14. It is interesting to clarify how the pair correlation in these exotic nuclei is different from that in stable nuclei, reflecting the strong density dependence mentioned above. In this connection we would like to ask how the pair correlation at low nucleon density is different from that around the normal density.

Spatial structure of the neutron Cooper pair is focused upon as a characteristic feature of the low density nucleon pairing. Its possible indication could be the di-neutron correlation in the two-neutron halo nuclei, e.g. ${ }^{11} \mathrm{Li}$, for which a spatially correlated pair formed by the halo neutrons has been predicted theoretically 8, 15, 16, 17, 18, 19, 20. and debated in experimental studies 21, 22, 23, 24]. Further, a recent theoretical analysis 25] using the Hartree-FockBogoliubov (HFB) method [26, 27, 28, 29] predicts also presence of similar di-neutron correlation in medium-mass neutron-rich nuclei where more than two weakly-bound neutrons contribute to form the neutron skin in the exterior of the nuclear surface. It is also possible to argue importance of the spatial correlation from a more fundamental viewpoint based on the nucleon interaction in the ${ }^{1} S$ channel. The bare nucleon-nucleon interaction in this channel has a virtual state around zero energy characterized by the large scattering length $a \approx-18 \mathrm{fm}$, which implies a very strong attraction between a pair of neutrons with the spin singlet configurations. A rather general argument [30, 31], which applies to a dilute limit of any Fermion systems, indicates that the pair correlation of the Fermions interacting with a large scattering length differs largely from what is considered in the conventional BCS theory 32 assuming weak coupling: it is then appropriate to consider a crossover between a superfluid system of the weak-coupling BCS type and a Bose-Einstein condensate of spatially compact bound Fermion pairs 30, 31, 33, 34, 35]. This BCS-BEC crossover phenomenon was recently observed in a ultra-cold atomic gas in a trap for which the interaction is controllable 36 . In the case of the nucleon pairing, the BCS-BEC crossover has been argued mostly for the neutron-proton pairing in the ${ }^{3} S D_{1}$ channel, for which the strong spatial correlation associated with the deuteron and the BEC of the deuterons may emerge 37, 38, 39, 40, 41]. Concerning the neutron pairing in the ${ }^{1} S$ channel, which we discuss in the present paper, we may also expect that the strong coupling feature may lead to the spatial di-neutron correlation although the realization of the crossover could be marginal and depend on the density [38].

In the present paper, we would like to clarify how the the spatial structure of the neutron Cooper pair varies with the density. For this purpose, we shall investigate the neutron pair correlation in symmetric nuclear matter and neutron matter in the low density region. Uniform matter is of course a simplification of the actual nucleon configurations in finite nuclei and neutron stars. However they have a great advantage as one can solve the gap equation in this case without ambiguity for various interactions including the bare nucleon-nucleon forces with the repulsive core 33, 4, 5, 39, 40, 41, 42, 43, 44, 45, 46, 47, 48, 49] as well as effective interactions such as the Gogny 
force 48, 50, 51], provided that the BCS approximation (equivalent to the HFB in finite nuclei) is assumed. It is straightforward then to determine the wave function of the neutron Cooper pair from the solution of the gap equation 3, 39, 40, 43, 44, 45, 47, 48. This provides us with a good reference frame to study the spatial structure of the Cooper pair as a function of the density while we do not intend to make precise predictions on other properties of neutron and symmetric nuclear matter. We shall perform an analysis employing both a bare force and the effective Gogny force [52], and using a Hartree-Fock single-particle spectrum associated with the media. Our main conclusion will be that the spatial di-neutron correlation is strong in a wide range of the low density $\rho / \rho_{0} \approx 10^{-4}-0.5$, independently on the adopted forces. We shall clarify the nature of the strong spatial di-neutron correlation in terms of the BCS-BEC crossover model.

We shall also examine a possibility of phenomenological description of the spatially correlated neutron Cooper pair. Here we consider a contact force with a parametrized density dependent interaction strength, called often the density dependent delta interaction (DDDI) 8, 9, 10, 11, 53, 54, 55, 56, 57, 58]. The parameters of the DDDI need to be determined from some physical constraints. For example, the interaction strength has been constrained by conditions to reproduce the experimentally extracted pairing gap in finite nuclei [9, 10, 11, 54, 55, 56, 57], or the density-dependence of the neutron pairing gap in symmetric nuclear matter and the ${ }^{1} S$ scattering length 8 , 58 . It should be noted here that the contact force requires a cut-off energy, which needs to be treated as an additional model parameter. Concerning the cut-off parameter, attentions have been paid in the previous studies to convergence properties of the pairing correlation energy in finite nuclei 9], to the energy dependence of the phase shift [59], or to the renormalization with respect to the pairing gap 60, 61]. In the present paper we shall take a different approach to the cut-off, i.e., we investigate relevance of the cut-off parameter to the spatial structure of the neutron Cooper pair. It will be shown that the cut-off energy plays an important role to describe the strong spatial correlation at low density. Considering this as a physical constraint on the cut-off energy, we shall derive new parameter sets of the DDDI.

Preliminary results of this work are reported in Ref. 62$]$.

\section{FORMULATION}

\section{A. BCS approximation}

We describe the neutron pair correlation in uniform neutron matter and in uniform symmetric nuclear matter by means of the BCS approximation, which is equivalent to the Bogoliubov's generalized mean-field approach 26, 27]. One of the basic equations is the gap equation, which is written in the momentum representation as

$$
\begin{aligned}
& \Delta(p)=-\frac{1}{2(2 \pi)^{3}} \int d \boldsymbol{k} \tilde{v}(\boldsymbol{p}-\boldsymbol{k}) \frac{\Delta(k)}{E(k)}, \\
& E(k)=\sqrt{(e(k)-\mu)^{2}+\Delta(k)^{2}} .
\end{aligned}
$$

Here $\Delta(k)$ is the pairing gap dependent on the single-particle momentum $k$, while $e(k)$ and $E(k)$ are the single-particle and the quasiparticle energies. $\tilde{v}(\boldsymbol{p}-\boldsymbol{k})$ is the matrix element of the nucleon-nucleon interaction in the ${ }^{1} S$ channel. The gap equation needs to be solved together with the number equation

$$
\rho \equiv \frac{k_{F}^{3}}{3 \pi^{2}}=\frac{1}{(2 \pi)^{3}} \int d \boldsymbol{k}\left(1+\frac{e(k)-\mu}{E(k)}\right),
$$

which determines the relation between the neutron density $\rho$ (the Fermi momentum $k_{F}$ ) and the chemical potential $\mu$. The solution of these equations defines the ground state wave function of the BCS type and the static pairing properties at zero temperature for a given density $\rho$.

As the interaction acting in the ${ }^{1} S$ channel we shall adopt a bare nucleon-nucleon force, the G3RS force 63 , and the effective interaction given by Gogny 52 . The G3RS force is a local potential representation of the bare nucleon-nucleon interaction which is given by a superposition of three Gaussian functions:

$$
v(\boldsymbol{r})=\sum_{i} v_{i} e^{-r^{2} / \mu_{i}^{2}}
$$

One component represents a repulsive core with the height of $v_{1}=2000 \mathrm{MeV}$ and the range parameter $\mu_{1}=0.447$ $\mathrm{fm}$, while two other Gaussians with $v_{2,3}=-240,-5 \mathrm{MeV}$ and $\mu_{2,3}=0.942,2.5 \mathrm{fm}$ represent the attraction dominant for $1 \lesssim r \lesssim 3 \mathrm{fm}$ (see Fig 1). In spite of the simple three Gaussian representation, the G3RS reproduces rather well the ${ }^{1} S$ phase shift up to about $300 \mathrm{MeV}$ in the c.m. energy of the scattering nucleons. The associated scattering length 


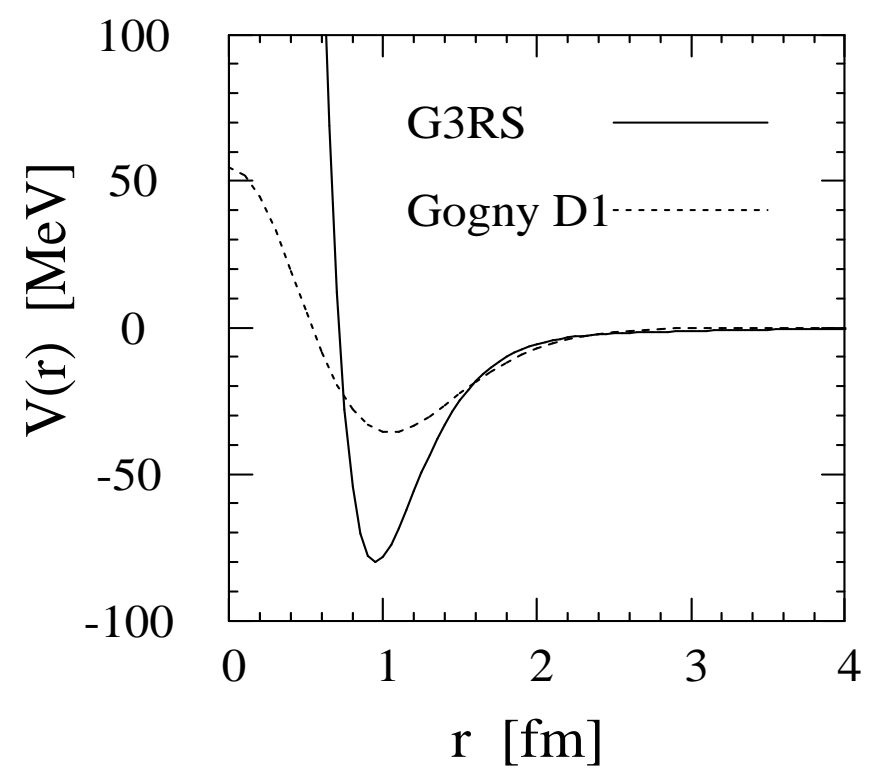

FIG. 1: The nucleon-nucleon potential $v(r)$ in the ${ }^{1} S$ channel for the G3RS and Gogny D1 forces, plotted with the solid and dotted curves, respectively, as a function of the relative distance $r$ between neutrons.

$a=-17.6 \mathrm{fm}$ is in close agreement with the experimental value $a=-18.5 \pm 0.4 \mathrm{fm} 64$. The G3RS has been used in some BCS calculations for the ${ }^{1} S$ pairing at low density and for the ${ }^{3} P_{2}$ pairing at high density [3, 4, [5]. From a practical point of view, the analytic form makes it easy to evaluate the matrix elements of the interaction.

The Gogny force is an effective interaction which is designed for the HFB description of the pairing correlation in finite nuclei while keeping some aspects of the $G$-matrix [52]. It is also a local potential represented as a combination of two Gaussian functions in the form of Eq.(4) with the range parameters $\mu_{1,2}=0.7,1.2 \mathrm{fm}$. In the following we show mostly results obtained with the parameter set D1 52] as we find no qualitative difference in the results for the parameter set D1S 65]. A common feature of the G3RS and Gogny forces is that both are attractive in the range $1 \lesssim r \lesssim 3 \mathrm{fm}$ while they differ largely for $r \lesssim 0.5 \mathrm{fm}$, where the Gogny force exhibits only a very weak repulsion instead of the short-range core present in the G3RS force (Fig (1). The interaction range of the two forces is of the order of $3 \mathrm{fm}$. Note that the experimental effective range is $r_{e}=2.80 \pm 0.11$ [64]. We shall also apply a zero-range contact force $v(\boldsymbol{r}) \propto \delta(\boldsymbol{r})$. Our treatment of this interaction will be described separately in Section $\nabla$

As the single-particle energy $e(k)$ we use, in the case of the Gogny interaction, the Hartree-Fock single-particle spectrum derived directly from the same interaction. In the case of the bare force, it would be better from a viewpoint of the self-consistency to use the Brueckner Hartree-Fock spectrum. But for simplicity we adopt in the present analysis an effective mass approximation. Namely the single-particle energy is given by $e(k)=k^{2} / 2 m^{*}$, where the effective mass $m^{*}=\left(\partial^{2} e(k) /\left.\partial^{2} k\right|_{k_{F}}\right)^{-1}$ is derived from the Gogny HF spectrum[50] for the parameter set D1.

We solve the gap and number equations, (11) and (3), without introducing any cut-off. The momentum integrations in the two equations are performed using a direct numerical method, where the maximum momentum $k_{\max }$ for the integration is chosen large enough so that the result does not depend on the choice of $k_{\max }$. We adopt $k_{\max }=20 \mathrm{fm}^{-1}$ for the G3RS, and $k_{\max }=10 \mathrm{fm}^{-1}$ for the Gogny interaction. Note here that it is dangerous to introduce a small energy window around the chemical potential (or the Fermi energy) or a cut-off at a small momentum in evaluating the r.h.s. of the gap equation. Such an approximation may be justified only in the case of the weak coupling BCS where the pairing gap is considerably smaller than the Fermi energy, but it is not applicable to the strong coupling case [30]. Note also that the chemical potential $\mu$ and the Fermi energy $e_{F}$ are not the same except in the limit of the weak coupling. We define the Fermi momentum $k_{F}$ through the nominal relation to the density $\rho=\frac{1}{3 \pi^{2}} k_{F}^{3}$, and the Fermi energy $e_{F}$ by $e_{F} \equiv e\left(k_{F}\right)$. The pairing gap $\Delta_{F} \equiv \Delta\left(k_{F}\right)$ at the Fermi momentum is used below as a measure of the pair correlation. In the following $\rho$ denotes always the neutron density. (The total nucleon density is $\rho_{\text {tot }}=2 \rho$ in the case of symmetric nuclear matter.) We define, as a reference value, the normal neutron density by $\rho_{0}=\frac{1}{3 \pi^{2}} k_{F 0}^{3}$ with $k_{F 0}=1.36 \mathrm{fm}^{-1}$.

In order to investigate the spatial structure of the neutron Cooper pair, it is useful to look into its wave function represented as a function of the relative distance between the partner neutrons of the pair. It is given by

$$
\Psi_{\text {pair }}(r) \equiv C^{\prime}\left\langle\Phi_{0}\left|\psi^{\dagger}(\boldsymbol{r} \uparrow) \psi^{\dagger}\left(\boldsymbol{r}^{\prime} \downarrow\right)\right| \Phi_{0}\right\rangle=\frac{C}{(2 \pi)^{3}} \int d \boldsymbol{k} u_{k} v_{k} e^{i \boldsymbol{k} \cdot\left(\boldsymbol{r}-\boldsymbol{r}^{\prime}\right)}
$$




$$
u_{k} v_{k}=\frac{\Delta(k)}{2 E(k)}
$$

in terms of the $u, v$-factors except by the normalization factors $C$ and $C^{\prime}$. Here $\psi^{\dagger}(\boldsymbol{r} \sigma) \quad(\sigma=\uparrow, \downarrow)$ is the creation operator of neutron and $\left|\Phi_{0}\right\rangle$ is the BCS ground state. The Cooper pair wave function depends only on the relative distance $r=\left|\boldsymbol{r}-\boldsymbol{r}^{\prime}\right|$ between the partners as it is an s-wave. We evaluate the momentum integral in Eq.(5) in the same way as in the gap and the number equations.

It is useful to evaluate the size of the neutron Cooper pair. A straightforward measure is the r.m.s. radius of the Cooper pair

$$
\xi_{r m s}=\sqrt{\left\langle r^{2}\right\rangle}
$$

where

$$
\left\langle r^{2}\right\rangle=\int d \boldsymbol{r} r^{2}\left|\Psi_{\text {pair }}(r)\right|^{2}=\frac{\int_{0}^{\infty} d k k^{2}\left(\frac{\partial}{\partial k} u_{k} v_{k}\right)^{2}}{\int_{0}^{\infty} d k k^{2}\left(u_{k} v_{k}\right)^{2}}
$$

can be calculated directly from the Cooper pair wave function $\Psi_{\text {pair }}(r)$ and/or from the $u$, $v$-factors in the momentum space. If one assumes weak coupling, the Pippard's coherence length [32]

$$
\xi_{P}=\frac{\hbar^{2} k_{F}}{m^{*} \pi \Delta_{F}}
$$

given analytically in terms of the gap and the Fermi momentum may be used also as another estimate of the size of the Cooper pair. In the following we mostly use $\xi_{r m s}$ since this quantity itself has a solid meaning even in the case of the strong coupling BEC case and in the crossover region between BCS and BEC. We shall use $\xi_{P}$ for qualitative discussions.

In the present paper, we neglect higher order many-body effects which go beyond the BCS approximation. In many calculations $66,67,68,69,70,71,72,73,74,75]$ the higher order effects in low density neutron matter are predicted to reduce the pairing gap by about a factor of two, which is however very much dependent on the prescriptions adopted [4, 5] except for the low density limit $\rho \rightarrow 0$ [76]. A recent Monte Carlo study [77] using the realistic bare force suggests the gap close to the BCS result. The higher order effects in symmetric nuclear matter 75] and in finite nuclei 78, 79, 80] are estimated to increase the gap. Keeping in mind these ambiguities, we consider that the BCS approximation provides a meaningful zero-th order reference.

\section{B. Pairing gap and coherence length}

Figure 2 shows the neutron pairing gap $\Delta_{F}$ obtained with the G3RS and Gogny D1 forces both for neutron matter and for symmetric matter. Results assuming the free single-particle spectrum (equivalent to the use of $m^{*}=m$ ) are also shown for comparison. The pairing gap becomes maximum around $\rho / \rho_{0} \sim 0.1-0.3$ in all the cases. The gap decreases gradually with further decreasing the density. The difference between neutron matter and symmetric nuclear matter, which originates from the effective mass effect, becomes negligible at low density $\rho / \rho_{0} \lesssim 5 \times 10^{-2}$.

The pairing gap obtained with the G3RS force is very similar to those obtained with more realistic models of the bare force (OPEG 3, 42, 43], Reid 3, 43, 46], Argonne 44, 46], Paris 44], Bonn 45, 48], Nijmegen 4]). This is because the gap is essentially determined by the ${ }^{1} S$ phase shift function $[81]$, and the G3RS force reproduces the experimental phase shift, though not as accurately as the modern forces. There is small difference from them: the maximum gap $\Delta_{F} \approx 2.5 \mathrm{MeV}$ around $\rho / \rho_{0} \sim 0.2\left(\right.$ or $k_{F} \sim 0.8 \mathrm{fm}^{-1}$ ) for neutron matter is slightly smaller in the G3RS by about 5-20\%. The difference may be due to a limitation of the simple three-Gaussian representation, but the quality is enough for the following discussions. The gap obtained with the Gogny force is consistent with those in the previous calculations [4, 50, 51]. If we compare with the Gogny and G3RS results, they exhibit a similar overall density dependence, and a significant difference between the two forces is seen only at modest density $\rho / \rho_{0} \gtrsim 5 \times 10^{-2}$. Garrido et al. [58] suggests that the similarity may indicate a possible cancellation among higher order effects in the case of symmetric matter. We find a less significant difference in the gap at rather low density $\rho / \rho_{0} \lesssim 10^{-3}$, though not visible in Fig 2. This arises from the fact that the scattering length $a=-13.5$ fm of the Gogny D1 force deviates from the G3RS value $a=-17.6 \mathrm{fm}$.

The r.m.s. radius $\xi_{r m s}$ of the neutron Cooper pair calculated for neutron and symmetric nuclear matter with the bare or the Gogny forces are shown in Fig 3 and Table The calculated $\xi_{r m s}$ is consistent with the r.m.s. radius (or the Pippard's coherence length) reported in the BCS calculations using other models of the bare force 3, 43, 47, 48]. 


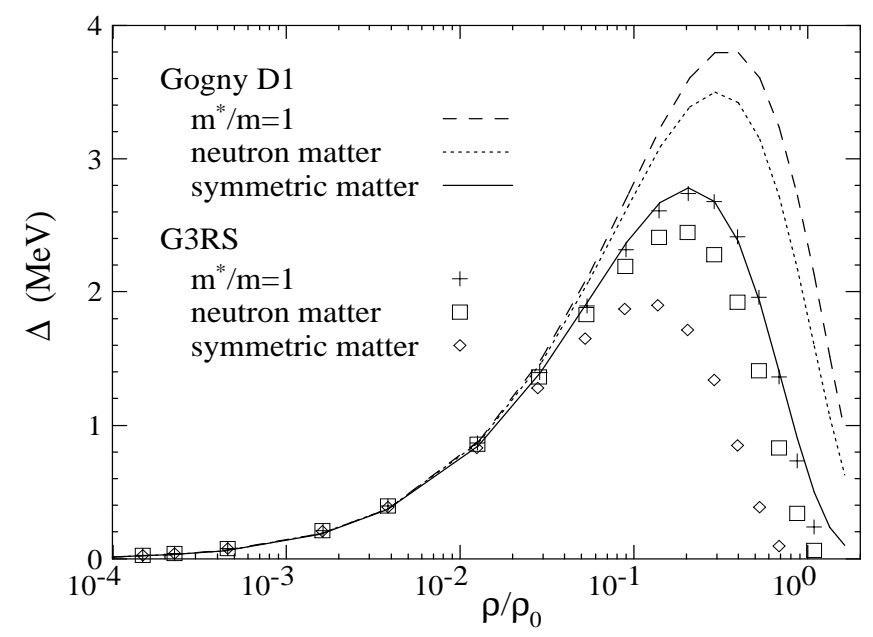

FIG. 2: The pairing gap $\Delta_{F}$ in neutron and symmetric nuclear matter as a function of the neutron density $\rho / \rho_{0}$. The results for the G3RS force are shown with the symbols: cross for the free single-particle spectrum, square for neutron matter, and diamond for nuclear matter. The results with the Gogny D1 force are plotted with the dashed, dotted, and solid curves for matter with the free single-particle spectrum, for neutron matter, and for symmetric matter, respectively.

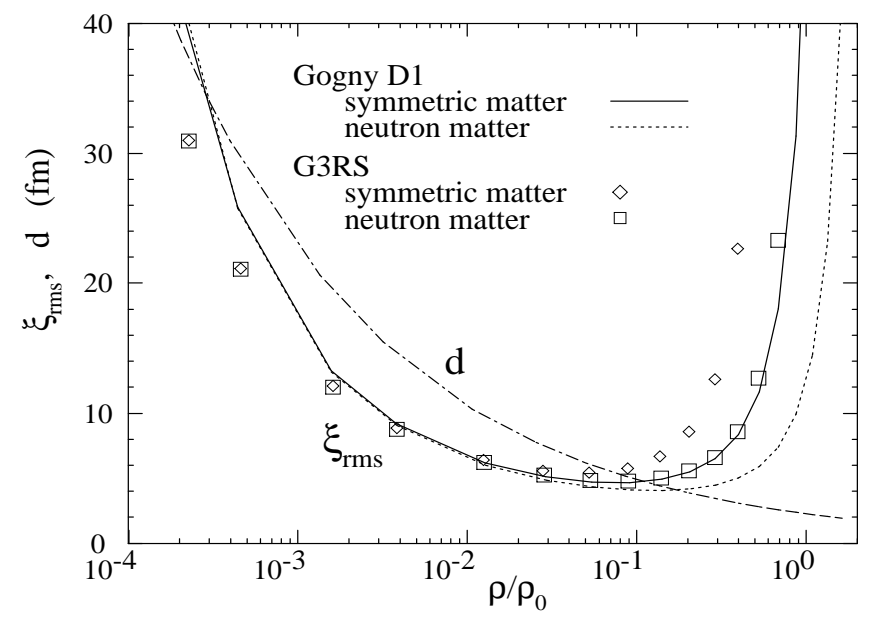

FIG. 3: The r.m.s. radius $\xi_{r m s}$ of the neutron Cooper pair in uniform matter, plotted as a function of the neutron density $\rho / \rho_{0}$. The results for symmetric nuclear and neutron matter obtained with the Gogny D1 force are shown by the solid and dotted curves, respectively, while the results for symmetric nuclear and neutron matter with the G3RS force are shown by the diamond and square symbols, respectively. The average inter-neutron distance $d=\rho^{-1 / 3}$ is plotted with the dot-dashed line.

Here we would like emphasize characteristic density dependence of $\xi_{r m s}$. It is seen from Fig B that $\xi_{r m s}$ decreases dramatically by nearly a factor of ten from a large value of the order of $\sim 50 \mathrm{fm}$ around the normal density $\rho / \rho_{0} \sim 1$ to considerably smaller values $\xi_{r m s} \approx 4.5-6 \mathrm{fm}$ at density around $\rho / \rho_{0} \sim 0.1$. The size of the Cooper pair stays at small values $\xi_{r m s} \approx 5-6 \mathrm{fm}$ in the density region $\rho / \rho_{0} \sim 10^{-2}-0.1$. It then turns to increase, but only gradually, at further low density. These features are commonly seen for both neutron and symmetric nuclear matter, and for both the G3RS and Gogny forces.

We would like to emphasize also the smallness of the neutron Cooper pair. This may be elucidated if we compare the r.m.s. radius $\xi_{r m s}$ with the average inter-neutron distance $d \equiv \rho^{-1 / 3}=3.09 k_{F}^{-1}$. It is seen from Fig 3 that $\xi_{r m s}$ becomes smaller than $d$ in a very wide range of density $\rho / \rho_{0} \sim 10^{-4}-0.1$. The ratio $\xi_{r m s} / d$ can reach values as small as $\approx 0.5$ at density around $\rho / \rho_{0} \sim 10^{-2}$. The relation $\xi_{r m s}<d$, i.e. the size of the neutron Cooper pair smaller than the average inter-neutron distance, suggests that the neutron Cooper pair exhibits strong spatial di-neutron correlation.

To understand this strong spatial correlation, it is useful to consider the ratio $\Delta_{F} / e_{F}$ between the pairing gap and the Fermi energy rather than the absolute magnitude of the gap. The pairing gap $\Delta_{F} \approx 0.2 \mathrm{MeV}$ at the density $\rho / \rho_{0}=1 / 512$ for example appears small in the absolute scale, but the gap to Fermi-energy ratio amounts to $\Delta_{F} / e_{F} \approx 0.4$ (see Table 【), which is larger than the value $\Delta_{F} / e_{F} \approx 0.25$ at $\rho / \rho_{0}=1 / 8$ where the gap is nearly 
the maximum. If we use the Pippard's coherence length $\xi_{P}=\hbar^{2} k_{F} / m^{*} \pi \Delta_{F}$ in place of the r.m.s. radius $\xi_{r m s}$ (this may be justified at least for qualitative discussion since the two quantities agree within 10-25\%, see Table प), the ratio between the r.m.s. radius and the average inter-neutron distance is related to the gap to Fermi-energy ratio as $\xi_{r m s} / d \sim \xi_{P} / d \sim 0.2 e_{F} / \Delta_{F}$. Consequently we can expect in the zero-th order argument that the strong spatial correlation $\xi_{r m s} / d \lesssim 1$ emerges when the gap to Fermi-energy ratio is larger than $\Delta_{F} / e_{F} \gtrsim 0.2$. This is realized in the present calculations in the density range $\rho / \rho_{0} \sim 10^{-4}-0.1$. In the following we shall investigate in detail the spatial correlation in the neutron Cooper pair around this density range.

We shall comment here comparison with the ${ }^{3} S D_{1}$ neutron-proton pairing in symmetric nuclear matter. In this case the BCS pairing gap calculated with a realistic bare force (the Paris force) is of the order of $8 \mathrm{MeV}$ at maximum[49]. The r.m.s. radius $\xi_{r m s}$ of the neutron-proton Cooper pair is quite small, reaching to the minimum value $\xi_{r m s} \sim 2$ fm at density around $\rho / \rho_{0} \sim 0.2$ [40]. Consequently, the r.m.s. radius $\xi_{r m s}$ becomes considerably smaller than the average inter-particle distance $d$ in the density interval from $\rho / \rho_{0} \sim 0.5$ down to the zero density limit, where $\xi_{r m s}$ becomes identical to the r.m.s. radius of the deuteron [40]. In the case of the ${ }^{1} S$ neutron pairing, by contrast, the signature of the strong coupling $\xi_{r m s} \lesssim d$ is obtained in the wide but limited range of the density $\rho / \rho_{0} \sim 10^{-4}-0.1$. Apart from this difference, it is noted that the qualitative trend of the Cooper pair size, e.g. shrinking with increasing the density from the zero density limit, is similar to that discussed in the neutron-proton case [40].

\begin{tabular}{ccccccccccc}
\hline \hline$k_{F}$ & $\rho / \rho_{0}$ & $d$ & $m^{*} / m$ & $e_{F}$ & $\Delta_{F}$ & $\xi_{r m s}$ & $\xi_{P}$ & $P(d)$ & $\left(1 / k_{F} a\right)_{\xi}$ & $\left(1 / k_{F} a\right)_{\Delta}$ \\
\hline symmetric matter, Gogny D1 & & & & & & & & & \\
1.36 & 1 & 2.27 & 0.668 & 62.0 & 0.64 & 46.60 & 41.76 & 0.18 & -2.91 & -2.99 \\
1.079 & $1 / 2$ & 2.87 & 0.744 & 33.8 & 2.03 & 10.80 & 9.45 & 0.48 & -1.83 & -1.84 \\
0.68 & $1 / 8$ & 4.55 & 0.891 & 10.9 & 2.60 & 4.81 & 3.87 & 0.81 & -0.97 & -0.90 \\
0.34 & $1 / 64$ & 9.10 & 0.984 & 2.45 & 0.97 & 5.87 & 4.71 & 0.92 & -0.59 & -0.52 \\
0.17 & $1 / 512$ & 18.20 & 0.998 & 0.60 & 0.22 & 12.05 & 10.30 & 0.91 & -0.62 & -0.58 \\
\hline neutron matter &, G3RS & & & & & & & & & \\
1.36 & 1 & 2.27 & 0.905 & 42.4 & 0.14 & 159.8 & 144.1 & 0.09 & -3.70 & -3.70 \\
1.079 & $1 / 2$ & 2.87 & 0.925 & 26.1 & 1.52 & 11.61 & 10.13 & 0.47 & -1.88 & -1.85 \\
0.68 & $1 / 8$ & 4.55 & 0.969 & 9.89 & 2.37 & 4.94 & 3.90 & 0.80 & -0.99 & -0.90 \\
0.34 & $1 / 64$ & 9.10 & 0.995 & 2.41 & 0.98 & 5.90 & 4.61 & 0.92 & -0.60 & -0.50 \\
0.17 & $1 / 512$ & 18.20 & 0.999 & 0.60 & 0.24 & 11.16 & 9.30 & 0.92 & -0.55 & -0.50 \\
\hline \hline
\end{tabular}

TABLE I: The pairing gap $\Delta_{F}$, the r.m.s. radius $\xi_{r m s}$, the Pippard's coherence length $\xi_{P}$, and the probability $P(d)$ within the average inter-neutron distance $d$, associated with the neutron Cooper pair in symmetric nuclear matter obtained with the Gogny D1 interaction and in neutron matter with the G3RS force, at the neutron density $\rho / \rho_{0}=1,1 / 2,1 / 8,1 / 64,1 / 512$, or equivalently $k_{F}=1.36,1.094,0.68,0.34,0.17 \mathrm{fm}^{-1}$. The Fermi energy $e_{F}$, the effective mass $m^{*} / m$, and the average interneutron distance $d$ are also shown. The parameters $\left(1 / k_{F} a\right)_{\xi}$ and $\left(1 / k_{F} a\right)_{\Delta}$ of the regularized delta interaction model are also listed (see the text). The units for $k_{F}$ and $\Delta_{F}, e_{F}$ are $\mathrm{fm}^{-1}$ and $\mathrm{MeV}$, respectively while that for $d, \xi_{r m s}$ and $\xi_{P}$ is fm.

\section{SPATIAL STRUCTURE OF NEUTRON COOPER PAIR}

\section{A. Cooper pair wave function: basics}

In examining the spatial structure of the neutron Cooper pairs, we shall focus mostly on the symmetric nuclear matter case obtained with the Gogny force and the neutron matter case with the G3RS force. The r.m.s. radii in these two cases represent a rough mean value of the four results plotted in Fig 3 Note also that the two r.m.s. radii coincide with each other within $10 \%$ in a very wide interval of density $\rho / \rho_{0}=10^{-3}-0.5$. It is by accident, but this feature can be exploited to single out influences of different interactions since the comparison can be made with the r.m.s. radii kept the same.

Figures 4 (a-e) show the wave function $\Psi_{\text {pair }}(r)$ of the neutron Cooper pair for the representative values of density listed in Table【 The result for neutron matter calculated with the G3RS force and that for symmetric nuclear matter with the Gogny D1 force are plotted in the same figure for the reasons mentioned just above. The probability density

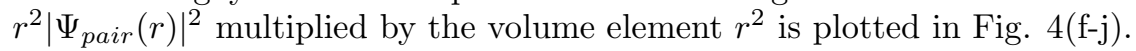

As a quantitative measure of the spatial correlation, we evaluate also the probability $P(r)$ for the partners of the neutron Cooper pair to come close with each other within a relative distance $r$. It is nothing but a partial integration 

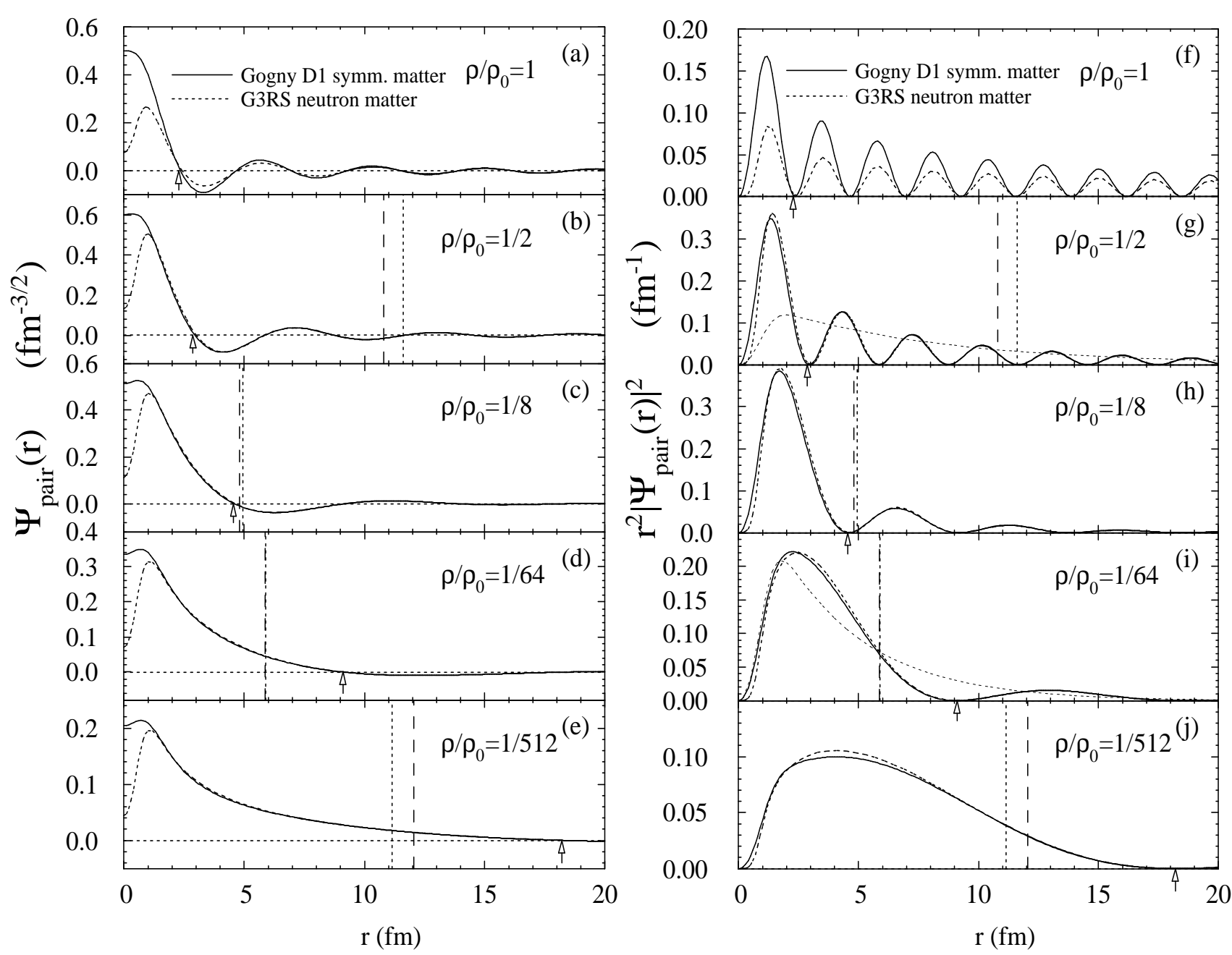

FIG. 4: (a-e) The wave function $\Psi_{\text {pair }}(r)$ of the neutron Cooper pair as a function of the relative distance $r$ between the pair partners at the neutron density $\rho / \rho_{0}=1,1 / 2,1 / 8,1 / 64,1 / 512$. The solid curve is for the pair in symmetric nuclear matter obtained with the Gogny D1 force, while the dotted curve is for that in neutron matter with the G3RS. The vertical dotted line represents the r.m.s. radius $\xi_{r m s}$ of the Cooper pair in neutron matter with the G3RS while the dashed line for symmetric nuclear matter with Gogny D1. Here and also in the following figures the wave function is normalized by $\int_{0}^{\infty}\left|\Psi_{\text {pair }}(r)\right|^{2} r^{2} d r=1$. The arrow indicates the average inter-neutron distance $d$. (f-j) The same as (a-e) but for the probability density $r^{2}\left|\Psi_{\text {pair }}(r)\right|^{2}$. The thin dotted line in (g) and (i) is the wave function of the fictitious "bound state" in the free space described in the text.

of the probability density $r^{2}\left|\Psi_{\text {pair }}(r)\right|^{2}$ up to the distance $r$ :

$$
P(r)=\frac{\int_{0}^{r}\left|\Psi_{\text {pair }}\left(r^{\prime}\right)\right|^{2} r^{\prime 2} d r^{\prime}}{\int_{0}^{\infty}\left|\Psi_{\text {pair }}\left(r^{\prime}\right)\right|^{2} r^{\prime 2} d r^{\prime}}
$$

An example of this quantity is shown in Fig $\left[\right.$ in the case of $\rho / \rho_{0}=1 / 2$.

Before proceeding to the main analysis we shall first point out that the G3RS and Gogny forces provide essentially the same spatial structure of the Cooper pair except at very short relative distances. In Fig 4 a clear difference between the two forces is seen at short relative distances $r \lesssim 1 \mathrm{fm}$. (Note that the normal density case, Fig प(a,f), is not relevant for this discussion since the gaps and the r.m.s. radii are very different.) Apparently the suppression of the wave function seen at $r \lesssim 1 \mathrm{fm}$ in the G3RS case is caused by the strong repulsive core present in the bare force. The Cooper pair wave function for the Gogny force does not show this short range correlation because of the lack of the core. On the other hand, by looking at distances $r>1 \mathrm{fm}$ slightly larger than the core radius we find that the Cooper pair wave functions obtained with the two forces agree quite well with each other. This observation applies also to the probability density $r^{2}\left|\Psi_{\text {pair }}(r)\right|^{2}$ and the probability $P(r)$, for which the difference at short distances $r<1 \mathrm{fm}$ becomes barely visible as the volume element is small at such short distances. Thus the spatial structure of the neutron Cooper pair does not depend on whether the interaction is the bare force or the effective Gogny force, 


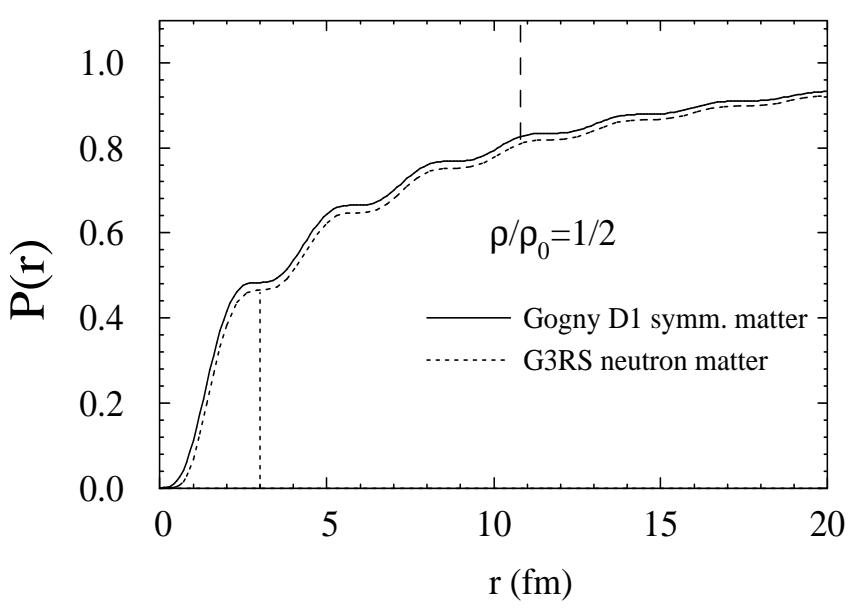

FIG. 5: The probability $P(r)$ for the partner neutrons of the Cooper pair to be correlated within a relative distance $r$, calculated at density $\rho / \rho_{0}=1 / 2$. The result for symmetric nuclear matter with the Gogny D1 force is plotted with the solid curve while the dotted curve represents the result for neutron matter with the G3RS force. The dashed vertical line indicates the r.m.s. radius of the Cooper pair in the symmetric matter case. The vertical dotted line marks the position $r=3$ fm.

provided that two cases gives the same r.m.s. radius of the Cooper pair. In the following, we shall concentrate on behaviors which are common to the two interactions.

The Cooper pair wave function $\Psi_{\text {pair }}(r)$ in the coordinate representation is reported in some of the previous BCS calculations adopting other models of the bare force 43, 44, 45, 47, 48] and the Gogny force 48]. Our wave function appears consistent with those in Refs. 43, 44, 45, 48] although in these references the wave function is shown only at very limited numbers of density values and up to not very large relative distances. We found, however, that the shape of the Cooper pair wave function shown in Ref. 47] differs largely from our results (Fig 4), especially at relative distances smaller than several fm.

\section{B. Density dependence}

If we compare in Fig 4 the Cooper pair wave functions at different density values, important features show up. An apparent observation is that the spatial extension or the size of the Cooper pair varies strongly with the density, in accordance with the strong density dependence of the r.m.s radius $\xi_{r m s}$ discussed in the previous section (Fig (3). We emphasize here another prominent feature. Namely the profile of the Cooper pair wave function also changes significantly with the density.

At the normal density $\rho / \rho_{0}=1$ (Fig $\left.4(\mathrm{a}, \mathrm{f})\right)$, the Cooper pair wave function is spatially extended: the r.m.s. radius of the Cooper pair is as large as $\xi_{r m s} \gtrsim 50 \mathrm{fm}$. The profile of the Cooper pair wave function in this case exhibits an exponential fall-off convoluted with an oscillation. This behavior is consistent with the well known expression 32 $r \Psi_{\text {pair }}(r) \sim K_{0}\left(r / \pi \xi_{P}\right) \sin \left(k_{F} r\right)$ for the Cooper pair wave function in the weak coupling BCS situation. Here $K_{0}$ is the modified Bessel function, which behaves asymptotically as $K_{0}\left(r / \pi \xi_{P}\right) \sim\left(\xi_{P} / r\right)^{1 / 2} \exp \left(-\left(r / \pi \xi_{P}\right)\right)$. The position of the first node $r \approx \pi k_{F}^{-1}$ approximately corresponds to the average inter-particle distance $d=3.09 k_{F}^{-1}(=2.3 \mathrm{fm})$.

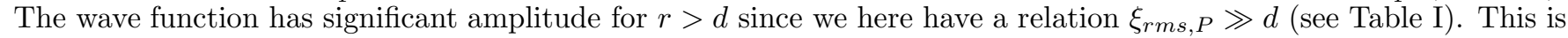
a typical behavior in the situation of the weak coupling BCS.

The Cooper pair wave function at the density $\rho / \rho_{0}=1 / 8$ (Fig $\left.4(\mathrm{c}, \mathrm{h})\right)$ is very different from that at the normal density. Apart from the considerably small spatial extension $\left(\xi_{r m s}=4.8-4.9 \mathrm{fm}\right)$, the functional form of the wave function behaves quite differently. We find that amplitude of the wave function is strongly concentrated within the average inter-neutron distance $d$, and that the oscillating amplitude beyond $d$ is quite small. This is consistent with the observation in the previous section that the r.m.s. radius $\xi_{r m s}$ of the Cooper pair is smaller than the average

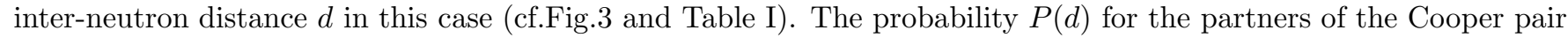
to be correlated within the inter-nucleon distance $d$, exceeds 0.8 (Fig [6] and Table \), indicating directly the strong spatial di-neutron correlation.

The neutron Cooper pair wave functions at $\rho / \rho_{0}=1 / 64$ and $1 / 512$, Fig प $(\mathrm{d}, \mathrm{e}, \mathrm{i}, \mathrm{j})$, exhibit a behavior similar to that at $\rho / \rho_{0}=1 / 8$. Inspecting more closely, we notice that the concentration within $r<d$ is stronger than at $\rho / \rho_{0}=1 / 8$ while the spatial extension itself is slightly larger $\left(\xi_{r m s} \approx 6-12 \mathrm{fm}\right)$. We observe also smaller oscillating amplitude in the large distance region $r>d$ (Fig प), larger values of $P(d) \approx 0.9$, and smaller ratio $\xi_{r m s} / d$ (Table प). They all 
point to stronger spatial di-neutron correlation at these values of density.

The Cooper pair wave function at $\rho / \rho_{0}=1 / 2(\operatorname{Fig} 4(\mathrm{~b}, \mathrm{~g}))$ exhibits an intermediate feature between that at the normal density $\rho / \rho_{0}=1$ and those at $\rho / \rho_{0}=1 / 64-1 / 512$. In particular, we notice that the spatial correlation seen at the lower density persists to some significant extent also in this case. For example, the probability density is strongly concentrated to the short distance region of $r \lesssim 3 \mathrm{fm}($ Fig $4(\mathrm{~g}))$. This is more apparent in the plot of $P(r)$ shown in Fig [5] where we find that the probability $P(r)$ increases steeply with increasing $r$ from $r=0$, and reaches $\sim 50 \%$ already at $r=3 \mathrm{fm}$, which is roughly the interaction range of the nucleon force. This strong concentration within $r<3$ fm may be elucidated by comparing with what could be expected if a bound pair having the same r.m.s. radius $\left(\xi_{r m s}=10.8 \mathrm{fm}\right.$ in this case) was formed in the free space. (We calculate this fictitious "bound state" wave function by increasing the strength of the Gogny D1 potential by a numerical factor.) It is noticed that the profile of the Cooper pair wave function differs from the "bound state" wave function which is plotted with the thin dotted line in Fig प (g). In this "bound state" wave function, concentration of the probability within $r \lessgtr 3 \mathrm{fm}$ is not very large, i.e., $P(3 \mathrm{fm})=0.24$, while the probability $P(3 \mathrm{fm})$ associated with the neutron Cooper pair wave function is about twice this value. This indicates that the spatial di-neutron correlation is also strong for the moderate low density region $\rho / \rho_{0} \sim 0.5$. A remnant of this spatial correlation is found also at the normal density (Fig $\left.4(\mathrm{a}, \mathrm{f})\right)$, but in this case the concentration within the interaction range is not very large $(P(3 \mathrm{fm})=0.21)$, due to the very large Cooper pair size $\left(\xi_{r m s} \sim 50 \mathrm{fm}\right)$. In contrast the Cooper pair wave function at the lower density, $\rho / \rho_{0}=1 / 64$ (Fig प 4 (i)) for example, is much more similar to the "bound state" wave function.

Figure [6 shows the overall behavior of $P(3 \mathrm{fm})$ and $P(d)$ as a function of the density. It is seen that the strong concentration within the interaction range, say $P(3 \mathrm{fm})>0.5$, is realized in the density region $\rho / \rho_{0} \approx 5 \times 10^{-2}-0.5$. The probability $P(3 \mathrm{fm})$ reaches the maximum value $\sim 0.7$ around $\rho / \rho_{0} \sim 0.1$, where the r.m.s. radius is the smallest. At lower density $\rho / \rho_{0} \lesssim 10^{-1}$, the probability $P(3 \mathrm{fm})$ decreases gradually in accordance with the gradual increase of the r.m.s. radius of the Cooper pair. Note however that in this density region $\left(\rho / \rho_{0} \sim 10^{-4}-10^{-1}\right)$ the concentration of the probability within the average inter-neutron distance $d$ remains very large, i.e., $P(d) \gtrsim 0.8$.

All the above analyses indicate that the spatial di-neutron correlation is strong in the quite wide density interval $\rho / \rho_{0} \sim 10^{-4}-0.5$.

It is interesting to compare our result with that in a similar analysis of the Cooper pair wave function for the ${ }^{3} S D_{1}$ neutron-proton pairing. In that case the Cooper pair wave function is found to merge smoothly into the deuteron wave function in the low density limit [39]. Correspondingly, the r.m.s. radius of the Cooper pair approaches to that of the deuteron, which is much smaller than the average inter-particle distance 40]. This is interpreted as a realization of the BEC of the deuterons in the low density region and the BCS-BEC crossover taking place with change of the density [38, 39, 40, 41]. In the neutron pairing case, the similarity of the Cooper pair wave function to a bound state wave function is found only in a limited density range $\rho / \rho_{0} \sim 10^{-4}-10^{-1}$, and it never converges to a bound state wave function (NB. there is no bound state in this channel in the free space). The r.m.s. radius $\xi_{r m s}$ of the Cooper pair is only comparable to the average inter-neutron distance $d$ in the same density interval. Although the spatial correlation is strong as discussed above, these qualitative observations alone are not enough to assess whether the region of the BCS-BEC crossover is reached in the case of the neutron pairing. We shall investigate this issue on more quantitative bases in the next section.

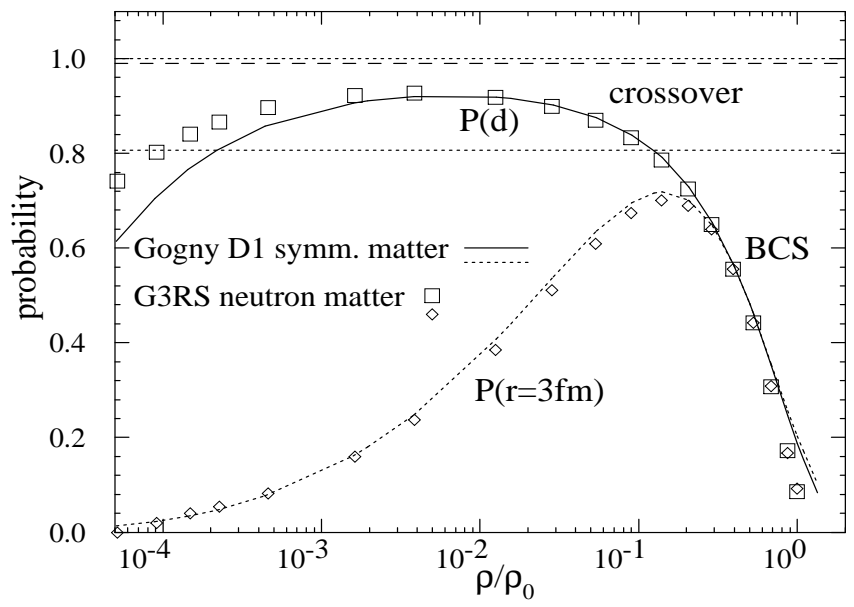

FIG. 6: The probability $P(d)$ for the partner neutrons to be correlated within the average inter-neutron distance $d$ and the probability $P(3 \mathrm{fm})$ within $r=3 \mathrm{fm}$. The solid and dotted curves are for symmetric nuclear matter obtained with the Gogny D1 force, while the square and diamond symbols are for neutron matter with the G3RS force. 


\section{RELATION TO THE BCS-BEC CROSSOVER}

In the previous section, we have seen the strong spatial correlation in the neutron Cooper pair wave function at low density. In the present section we shall elucidate its implication by making a connection to the BCS-BEC crossover phenomenon.

For this purpose, we shall first describe a reference Cooper pair wave function based on a simple solvable model of the BCS-BEC crossover, and then compare our results with that of the reference model. As such a reference, we adopt $a^{1} S$ pairing model that applies generically to a dilute gas limit of any Fermion systems [30, 33, 34], for which the average inter-particle distance $d=\rho^{1 / 3}$ is supposed to be much larger than the range of interaction. The dilute limit is equivalent to treating the interaction matrix elements as a constant, or assuming a contact interaction. Using the relation between the interaction constant and the zero-energy $T$-matrix or the scattering length $a$, the gap equation (11) is written in a regularized form:

$$
\frac{m}{4 \pi \hbar^{2} a}=-\frac{1}{2(2 \pi)^{3}} \int d \boldsymbol{k}\left(\frac{1}{E(k)}-\frac{1}{e(k)}\right) .
$$

The regularized gap equation (11) and the number equation (3) are now expressed analytically in terms of some special functions, and are easily solvable 82,83 . In this model a dimensionless parameter $1 / k_{F} a$, characterized by the scattering length and the Fermi momentum, is the only parameter that controls the strength of interaction, and hence properties of the pair correlation are determined solely by $1 / k_{F} a$ while the length scale is given by $k_{F}^{-1}$ (or the inter-particle distance $d=3.09 k_{F}^{-1}$ ), and the energy scale by the Fermi energy $e_{F}=\hbar^{2} k_{F}^{2} / 2 m[30,33$, 34, 35, 82]. The gap to Fermi-energy ratio $\Delta / e_{F}$ and the ratio $\xi_{r m s} / d$ between the r.m.s. radius and the average inter-particle distance are then monotonic functions of the interaction parameter $1 / k_{F} a[34,82$. The functional form of the Cooper pair wave function, defined by Eq.(5), is also determined only by $1 / k_{F} a$, except for the length scale. Since there is no available analytic expression for the wave function, we evaluate Eq.(5) by performing the momentum integral numerically with a help of an explicit use of a smooth cut-off function of a Gaussian form. The cut-off scale is chosen large enough so that the results shown below do not depend on it.

The range $1 / k_{F} a \ll-1$ of the interaction parameter corresponds to the situation of the weak coupling BCS, for which the pairing gap is given by the well known formula $4,30,35 \Delta / e_{F} \approx 8 e^{-2} \exp \left(\pi / 2 k_{F} a\right)$. In the opposite range $1 / k_{F} a \gg 1$, the situation of the Bose-Einstein condensation (BEC) of bound Fermion pairs (bosons) is realized. The crossover between the weak coupling BCS and the strong coupling BEC corresponds to the interval $-1 \lesssim 1 / k_{F} a \lesssim 1$, as described in Refs. [30, 33, 34, 35]. (The case with the infinite scattering length $1 / k_{F} a=0$ is the midway of the crossover, called the unitarity limit.) In the following we shall adopt $1 / k_{F} a= \pm 1$ according to Ref. 34] as boundaries characterizing the crossover although the transition is smooth in nature.

In Table II we list the values of $\xi_{r m s} / d$ and $\Delta_{F} / e_{F}$ at the "boundaries" $1 / k_{F} a= \pm 1$ of the crossover domain and at the unitarity limit $1 / k_{F} a=0$ [34, 82]. Note that the r.m.s. radius comparable to the average inter-particle distance $0.2 \lesssim \xi_{r m s} / d \lesssim 1.1$, or the gap comparable to the Fermi energy $0.2 \lesssim \Delta_{F} / e_{F} \lesssim 1.3$ corresponds to the BCSBEC crossover domain $-1 \lesssim 1 / k_{F} a \lesssim 1$. We have discussed in the previous section the probability $P(d)$ for the paired neutrons to come closer than the average inter-neutron distance $d$ (cf. Fig 6). As the same quantity is easily calculated also in the analytic model of the BCS-BEC crossover (the result is shown in Fig [8), this quantity may be used also as a measure of the crossover. The calculated boundary values corresponding to $1 / k_{F} a= \pm 1,0$ are listed in Table $\amalg$ The crossover region is specified by $0.8 \lesssim P(d) \lesssim 1.0$ while the boundary to the strong coupling $\mathrm{BEC}$ regime $(P(d) \approx 1)$ is hardly visible in this measure.

In the case of nucleonic matter, the assumption of the dilute gas limit may be justified only at very low density $\rho / \rho_{0} \lesssim 10^{-5}$ (or $k_{F} \lesssim 0.05 \mathrm{fm}^{-1}$ ) [4, 46, 84], and hence we cannot apply the above analytic model in a direct manner to the region of the density $\rho / \rho_{0}=10^{-5}-1$ which we are dealing with. In order to make the application possible, we shall stand on a more flexible viewpoint by regarding the interaction parameter $1 / k_{F} a$ as a freely adjustable variable, rather than by fixing it from the physical value of the neutron scattering length. We shall call the model treated in this way the regularized delta interaction model to distinguish from the original idea of the dilute gas limit.

The interaction parameter $1 / k_{F} a$ needs to be determined then. We shall require the condition that the regularized delta interaction model gives, for a given value of density, the same r.m.s. radius $\xi_{r m s} / d$ as that of the microscopically calculated neutron Cooper pair. The parameter determined in this way may be denoted $\left(1 / k_{F} a\right)_{\xi}$. We can also determine the interaction parameter to reproduce the ratio $\Delta_{F} / e_{F}$ between the gap and the Fermi energy, which we shall denote $\left(1 / k_{F} a\right)_{\Delta}$. The values of $\left(1 / k_{F} a\right)_{\xi}$ and $\left(1 / k_{F} a\right)_{\Delta}$ thus determined are listed in Table $\llbracket$ There is no sizable difference between $\left(1 / k_{F} a\right)_{\xi}$ and $\left(1 / k_{F} a\right)_{\Delta}$. The Cooper pair wave functions obtained in this reference model are shown in Fig 7 It is hard to distinguish between the two options of $1 / k_{F} a$. We now compare them with the neutron Cooper pair obtained with the Gogny force for symmetric matter at the three representative values of density $\rho / \rho_{0}=1,1 / 8$ and $1 / 512$. 

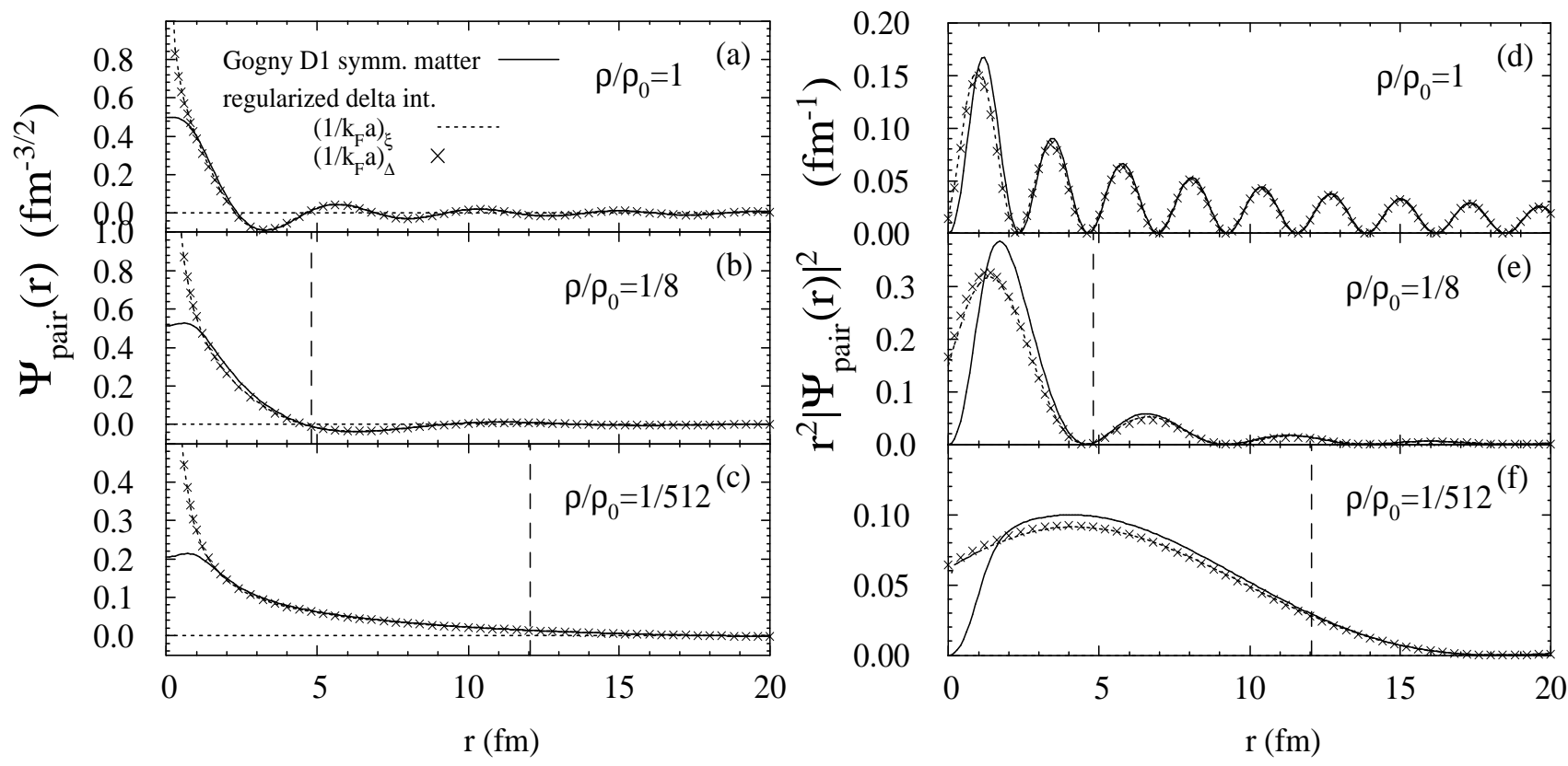

FIG. 7: (a-c) The neutron Cooper pair wave function $\Psi_{\text {pair }}(r)$ in the regularized delta interaction model, plotted with the dotted curve and the cross symbol in the cases of $\left(1 / k_{F} a\right)_{\xi}$ and $\left(1 / k_{F} a\right)_{\Delta}$, respectively. The neutron Cooper pair wave function in symmetric nuclear matter obtained with the Gogny D1 force is also shown by the solid curve. (d-f) The same as (a-c) but for the probability density $r^{2}\left|\Psi_{\text {pair }}(r)\right|^{2}$.

It is seen from Fig 7 that the wave function of the regularized delta interaction model and and that of the neutron Cooper pair behave very similarly at distances far outside the interaction range, $r \gtrsim 5$ fm. In contrast, we notice a sizable disagreement for $r \lesssim 3 \mathrm{fm}$. The disagreement is understandable as the wave function within the interaction range $r \approx 3 \mathrm{fm}$ of the finite range Gogny force could not be described by the zero-range delta interaction. The Cooper pair wave function in the regularized delta interaction model exhibits the known divergence $\Psi_{\text {pair }}(r) \propto 1 / r$ for $r \rightarrow 0$ (cf. Fig 7 (a-c)), and consequently the disagreement between the Gogny model and the regularized delta interaction model becomes serious at very short relative distances $r \lesssim 1 \mathrm{fm}$. Note however that the squared wave function weighted with the volume element $r^{2}$ stays finite as seen Fig 7 (d-f) and hence there is no diverging difference in the probability density. These observations suggest that the regularized delta interaction model can account for the essential features of the spatial structure of the neutron Cooper pair as far as the interaction strength $1 / k_{F} a$ is chosen appropriately.

\begin{tabular}{ccccc}
\hline \hline $1 / k_{F} a$ & $\xi_{r m s} / d$ & $\Delta / e_{F}$ & $P(d)$ & \\
\hline-1 & 1.10 & 0.21 & 0.807 & boundary to BCS \\
0 & 0.36 & 0.69 & 0.990 & unitarity limit \\
1 & 0.19 & 1.33 & 1.000 boundary to BEC \\
\hline \hline
\end{tabular}

TABLE II: The reference values of $1 / k_{F} a, \xi_{r m s} / d$ and $\Delta / e_{F}$ characterizing the BCS-BEC crossover in the regularized delta interaction model.

We thus have a reference frame, i.e., the regularized delta interaction model, to which the neutron pairing is mapped. The question on possible relation to the BCS-BEC crossover phenomena can be addressed now quantitatively. We first look into the ratio $\xi_{r m s} / d$ between the r.m.s radius $\xi_{r m s}$ of the Cooper pair and the average inter-particle distance $d$. The values of $\xi_{r m s} / d$ for the neutron Cooper pair obtained with the G3RS force and the Gogny D1 interaction are compared in Fig 9] with the reference values defining the "boundaries" of the BCS-BEC crossover domain (Table II). It is seen in Fig 9 that the calculated ratio $\xi_{r m s} / d$ enters the domain of the BCS-BEC crossover, $1.10>\xi_{r m s} / d(>0.19)$, in the density interval $\rho / \rho_{0} \approx 10^{-4}-0.1$. Note also the calculated ratio becomes closest to the unitarity limit $\xi_{r m s} / d=0.36$ around the density $\rho / \rho_{0} \sim 10^{-2}$. In the other way around, the weak coupling BCS regime is realized only at very low density $\rho / \rho_{0} \lesssim 10^{-4}$ and around the normal density $\rho / \rho_{0} \gtrsim 0.2$.

Comparing in Fig[10 the gap to Fermi-energy ratio $\Delta_{F} / e_{F}$ with the boundary values $0.21<\Delta_{F} / e_{F}<1.33$, we have 


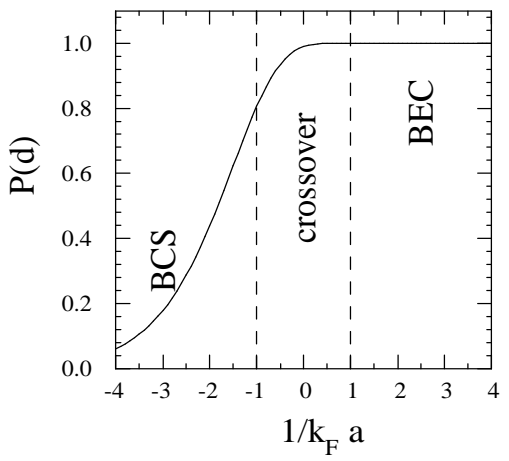

FIG. 8: The probability $P(d)$ for the partner particles to be correlated within the average inter-particle distance $d$ for the regularized delta interaction model.

the same observation that the density region $\rho / \rho_{0} \sim 10^{-4}-0.1$ corresponds to the domain of the BCS-BEC crossover. Comparison of the third measure $P(d)$, performed in Fig 6 provides us the same information. It is seen also in the values of $\left(1 / k_{F} a\right)_{\xi}$ and $\left(1 / k_{F} a\right)_{\Delta}$ listed in Table \that the condition of the crossover region $\left(1 / k_{F} a\right)_{\xi, \Delta}>-1$ is met in the cases of $\rho / \rho=1 / 8,1 / 64$ and $1 / 512$.

On the basis of the above analysis, we conclude that the strong spatial correlation at short relative distances seen in the neutron Cooper pair in the very wide density range $\rho / \rho_{0} \approx 10^{-4}-0.1$ is the behavior associated with the BCS-BEC crossover.

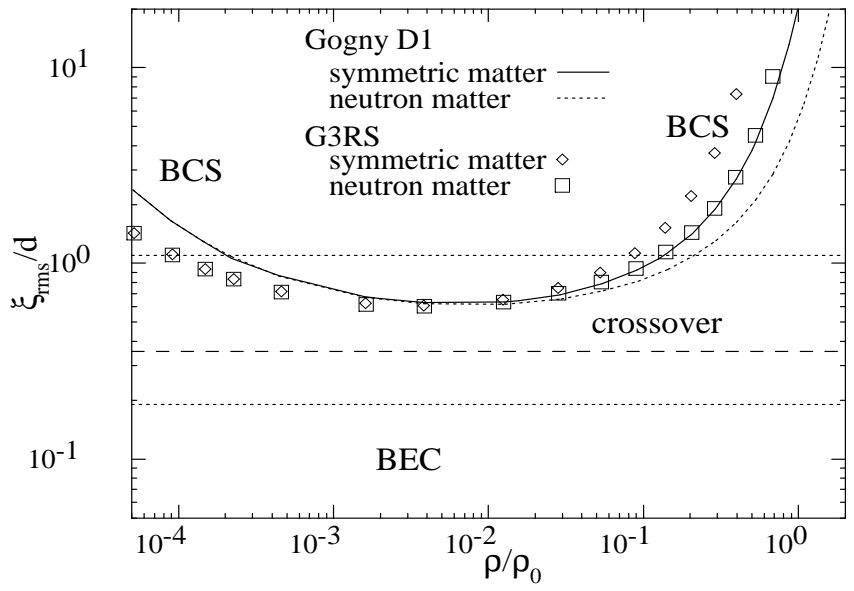

FIG. 9: The ratio $\xi_{r m s} / d$ between the r.m.s. radius $\xi_{r m s}$ of the neutron Cooper pair and the average inter-nucleon distance $d$, calculated with the Gogny D1 force for symmetric nuclear and neutron matter (the solid and dotted lines, respectively), and those with the G3RS force for symmetric nuclear and neutron matter (the diamond and square symbols), plotted as a function of the neutron density. The reference values characterizing the BCS-BEC crossover listed in Table II are also shown with the horizontal dotted and dashed lines.

\section{DENSITY-DEPENDENT DELTA INTERACTION}

\section{A. DDDI and the cut-off energy}

The contact force whose interaction strength is chosen as a density-dependent parameter, called often the densitydependent delta interaction (DDDI), has been employed as a phenomenological effective interaction describing the pairing correlation in finite nuclei, especially unstable nuclei with large neutron excess 9 , 10, 11, 25, 61, 85, 86, 87, 88]. In the previous section we found that the regularized contact interaction model, which employs also the contact force but with an analytic regularization, describes the essential feature of the spatial structure of the neutron Cooper pair wave function in the whole density range. This suggests a possibility that the phenomenological DDDI may also describe the spatial correlation in the neutron pairing. We would like to examine from this viewpoint in what conditions the DDDI can be justified. 


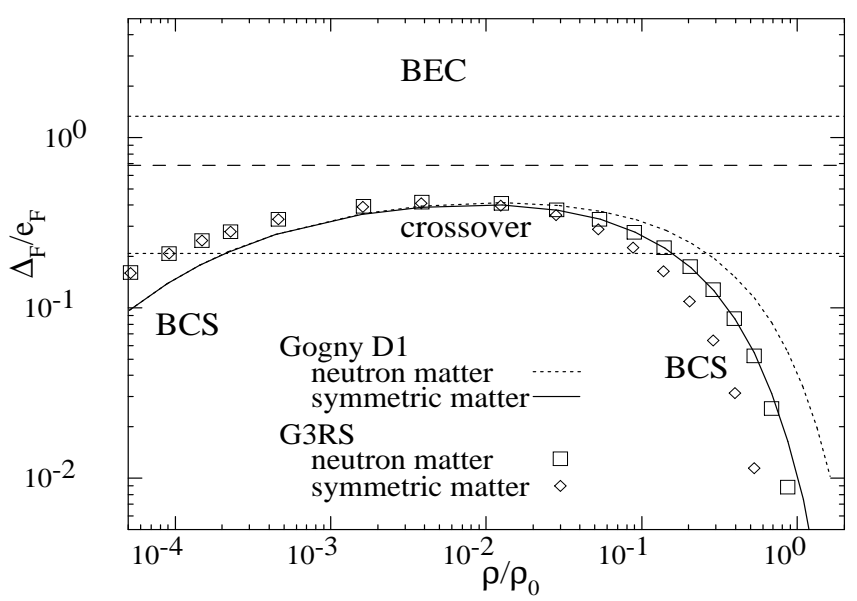

FIG. 10: The ratio $\Delta_{F} / e_{F}$ between the neutron pairing gap $\Delta_{F}$ and the neutron Fermi energy $e_{F}$, plotted as a function of the neutron density. The curves and the symbol are the same as in Fig 9 The reference values characterizing the BCS-BEC crossover listed in Table II are also shown with the horizontal lines.

The density-dependent delta interaction has a form

$$
v(\boldsymbol{r})=\frac{1-P_{\sigma}}{2} V_{0}[\rho] \delta(\boldsymbol{r}),
$$

where $V_{0}[\rho]$ is the interaction strength which is supposed to be dependent on the density. The force acts only in the ${ }^{1} S$ channel due to the projection operator $\left(1-P_{\sigma}\right) / 2$. It should be noted that in the applications of the density-dependent delta interaction to finite nuclei, an explicit and finite cut-off energy needs to be introduced. The cut-off energy in this case is regarded as an additional model parameter.

Applying the DDDI to the neutron pairing in uniform matter, the gap equation reads

$$
\Delta=-\frac{V_{0}}{2(2 \pi)^{3}} \int^{\prime} d \boldsymbol{k} \frac{\Delta}{E(k)}
$$

where the pairing gap here is a momentum independent constant $\Delta$. For the single-particle energy $e(k)$, we adopt the effective mass approximation. We use the effective mass derived from the Hartree-Fock spectrum of the Gogny D1. The momentum integral in Eq. (13) is performed under a sharp cut-off condition

$$
e(k)<\mu+e_{c u t}
$$

where we define the cut-off energy $e_{c u t}$ as relative energy from the chemical potential $\mu$. We shall treat $e_{c u t}$ as a common constant which is applied to all density. We use the same cut-off in calculating the Cooper pair wave function.

We remark that our definition of the cut-off is different from a similar one adopted in Refs. [8, [58], where a cut-off energy is defined with respect to the single-particle energy $e(k)$ measured from the bottom of the spectrum $e(k=0)$, e.g., by imposing $e(k)<60 \mathrm{MeV}[58]$ independent of the density. In our case, by contrast, we fix an energy window above the chemical potential $\mu$ to $e_{c u t}$. We think that the cut-off energy $e_{c u t}$ defined in this way can be compared with the quasiparticle energy cut-off $E_{i}<E_{c u t}$ adopted often in the HFB calculations for finite nuclei $\left(E_{i}\right.$ is the quasiparticle energy of the single-particle state $i)[9,10,11,25,28,61,85,86,87,88]$. Note that in finite nuclei the density varies locally with the position coordinate while the quasiparticle energy is defined globally. This may imply, in the sense of the local density approximation, that a fixed density-independent cut-off quasiparticle energy $E_{c u t}$ is applied to each value of local density. Note also that both our $e_{c u t}$ and the cut-off quasiparticle energy $E_{c u t}$ are quantities measured from the chemical potential, which approximately coincide if $e_{c u t}$ and $E_{c u t}$ are sufficiently larger than the pairing gap. Thus we can compare directly $e_{c u t}$ and $E_{c u t}$, provided that $e_{c u t}$ is chosen as a densityindependent constant. At the zero-density limit with $\mu=0$, the cut-off energy $e_{c u t}$ simply defines an upper bound on the free single-particle energy $e(k)=\hbar^{2} k^{2} / 2 m$.

\section{B. Constraints on $e_{c u t}$}

Let us investigate how the energy cut-off influences the Cooper pair wave function. We performed several calculations using different values of $e_{c u t}=5,10,30,50,100,200 \mathrm{MeV}$ for symmetric nuclear matter. In doing so, we choose the 
interaction strength $V_{0}$ for each value of density so that the gap $\Delta$ calculated with a given cut-off energy $e_{c u t}$ coincides with the gap $\Delta_{F}$ obtained with the Gogny D1 force. Note that the interaction strength $V_{0}$ thus determined depends on both the cut-off energy and the density.

\begin{tabular}{|c|c|c|c|c|c|c|c|}
\hline \multirow[b]{2}{*}{$\rho / \rho_{0}$} & \multirow[b]{2}{*}{$e_{\text {cut }}=5$} & \multicolumn{4}{|c|}{$\xi_{r m s}[\mathrm{fm}]$} & \multirow[b]{2}{*}{$200 \mathrm{MeV}$} & \multirow[b]{2}{*}{ Gogny D1 } \\
\hline & & 10 & 30 & 50 & 100 & & \\
\hline 1 & $\underline{48.6}$ & $\underline{47.3}$ & $\underline{46.7}$ & $\underline{46.6}$ & $\underline{46.5}$ & $\underline{46.5}$ & 46.6 \\
\hline $1 / 2$ & 16.9 & 13.8 & $\underline{11.2}$ & $\underline{10.9}$ & $\underline{10.7}$ & $\underline{10.6}$ & 10.8 \\
\hline $1 / 8$ & 14.9 & 10.0 & 6.0 & $\underline{5.3}$ & $\underline{4.8}$ & $\underline{4.6}$ & 4.8 \\
\hline $1 / 64$ & 10.5 & 7.9 & $\underline{6.2}$ & $\underline{6.0}$ & $\underline{5.7}$ & $\underline{5.5}$ & 5.9 \\
\hline $1 / 512$ & $\underline{13.2}$ & $\underline{12.5}$ & $\underline{12.0}$ & $\underline{11.9}$ & $\underline{11.8}$ & $\underline{11.7}$ & 12.1 \\
\hline
\end{tabular}

TABLE III: The r.m.s. radius $\xi_{r m s}$ of the neutron Cooper pair in symmetric nuclear matter obtained with the density dependent delta interaction and different cut-off energies $e_{c u t}=5,10,30,50,100,200 \mathrm{MeV}$. In the rightmost column, the r.m.s. radius for the Gogny D1 force is also listed. The underline means that the calculated number agrees with the reference Gogny D1 result within $10 \%$.
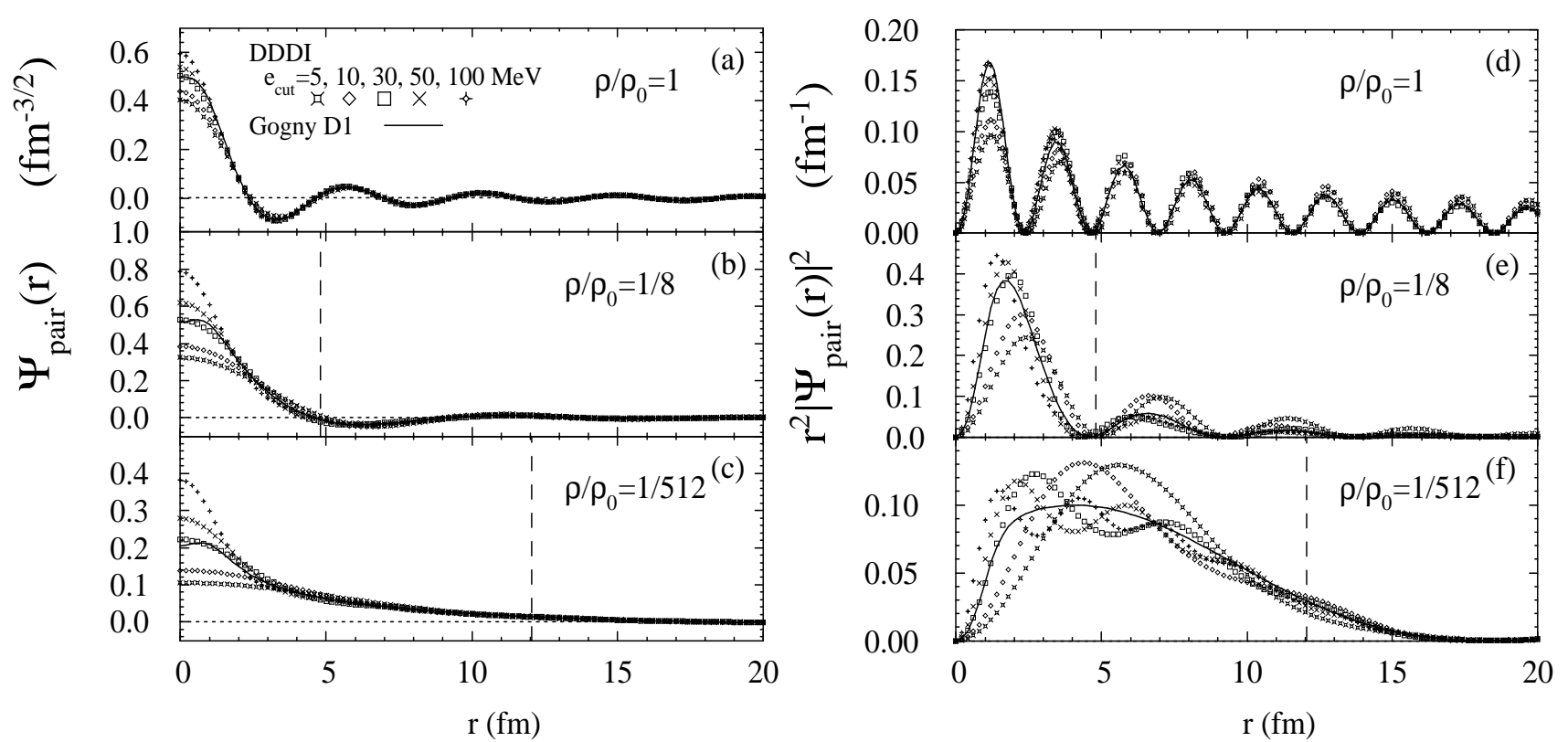

FIG. 11: (a-c) The neutron Cooper pair wave function $\Psi_{\text {pair }}(r)$ in symmetric nuclear matter calculated with the DDDI having different cut-off energies $e_{c u t}=5,10,30,50,100 \mathrm{MeV}$. The result obtained with the Gogny D1 interaction is shown also by the solid curve. (d-f) The same as (a-c) but for the probability density $r^{2}\left|\Psi_{\text {pair }}(r)\right|^{2}$.

Table III shows the r.m.s. radius $\xi_{r m s}$ of the neutron Cooper pair calculated with the DDDI for different values of the cut-off energy. Here $\xi_{r m s}$ is calculated by using the Cooper pair wave function in the coordinate representation evaluated up to $r=500 \mathrm{fm}$. It is seen from Table that the result apparently depends on the cut-off energy $e_{\text {cut }}$. In the cases of $\rho / \rho_{0}=1 / 64-1 / 2$, the dependence of $\xi_{r m s}$ on $e_{c u t}$ is very strong. The values calculated with the small cut-off energy $e_{\text {cut }}=5,10 \mathrm{MeV}$ largely deviate from those obtained with the Gogny force even though the interaction strength is chosen to reproduce the same reference pairing gap. We consider that the small cut-off energies $e_{c u t}=5,10$ $\mathrm{MeV}$ are unacceptable since they fail to describe the small size $\xi_{r m s} \sim 5 \mathrm{fm}$ of the neutron Cooper pair at the density around $\rho / \rho_{0}=10^{-2}-0.1$. If we require that the DDDI reproduces the r.m.s. radius of the neutron Cooper pair within an accuracy of $10 \%$ in the whole density region of interest, use of a large value of the cut-off energy satisfying $e_{\text {cut }} \gtrsim 50 \mathrm{MeV}$ is suggested.

To see roles of the cut-off energy in more details, we show in Fig 11 the neutron Cooper pair wave functions $\Psi_{\text {pair }}(r)$ obtained for $e_{c u t}=5,10,30,50,100 \mathrm{MeV}$. The plot of $\Psi_{\text {pair }}(r)$ indicates clearly that the Cooper pair wave function depends sensitively on the cut-off energy $e_{\text {cut }}$. If we adopt the small cut-off energies $e_{\text {cut }}=5,10 \mathrm{MeV}$ the wave function obtained with the DDDI fails to produce the strong spatial correlation at the short relative distances $r \lesssim 3$ 
$\mathrm{fm}$, which is the characteristic feature of the neutron Cooper pair wave function common to the Gogny and G3RS forces. (Fig [1] shows only the Gogny result for comparison, but we remind the reader of Fig 4 where the G3RS case is also shown.) The plot of the probability density $r^{2}\left|\Psi_{\text {pair }}(r)\right|^{2}$ at the density $\rho / \rho_{0}=1 / 8$ indicates that even the wave function at larger distances is not described well if the small cut-off energies $e_{c u t}=5,10 \mathrm{MeV}$ are adopted. This is nothing but the difficulty mentioned above in describing the r.m.s. radius with these small cut-off energies. If we use a large cut-off energy, say $e_{\text {cut }} \gtrsim 30-50 \mathrm{MeV}$, the wave function at large distances converges reasonably to that obtained with the Gogny force. Concerning the wave function at short relative distances $r \lesssim 3 \mathrm{fm}$, on the other hand, we find no convergence with respect to the cut-off energy. The value of the wave function $\Psi_{\text {pair }}(0)$ at zero relative distance $r=0$ increases monotonically with increasing $e_{c u t}$. (Increasing further $e_{c u t} \rightarrow \infty, \Psi_{\text {pair }}(r)$ will approach to the one for the regularized delta interaction model shown in Fig 7 and the value $\Psi_{\text {pair }}(0)$ at $r=0$ will diverge.)

It may be possible to regard $e_{c u t}$ as a parameter which simulates the finite range of the neutron-neutron interaction. It is then reasonable to require that the wave function $\Psi_{\text {pair }}(r)$ of the DDDI model with an appropriate choice of $e_{\text {cut }}$ describes that of the Gogny force at distances $r \lesssim 3 \mathrm{fm}$ (within the interaction range) as well as at larger distances. In the case of $\rho / \rho_{0}=1 / 8$, for example, this requirement is approximately satisfied if we choose $e_{\text {cut }}=30$ or $50 \mathrm{MeV}$, see Fig 111(b). At $\rho / \rho_{0}=1 / 512$, a good description of the wave function is obtained with $e_{\text {cut }}=30 \mathrm{MeV}$ (Fig [11(c)), and similarly we find $e_{c u t} \sim 70 \mathrm{MeV}$ for the normal density $\rho / \rho_{0}=1$ (Fig 11(a)). If we do not include in the comparison the wave function at very short distances $r \lesssim 1 \mathrm{fm}$ where the repulsion due to the core influences in the case of the bare force, the constraint on the cut-off energy may be slightly relaxed. For example, at the density $\rho / \rho_{0}=1 / 512$, the wave functions for $e_{c u t}=30$ and $50 \mathrm{MeV}$ differ only by about $\lesssim 20 \%$ at distances $1<r<3 \mathrm{fm}$, and hence the cut-off energy $e_{\text {cut }}=50 \mathrm{MeV}$ may also be accepted. Within this tolerance we can choose a value around $e_{\text {cut }} \sim 50$ $\mathrm{MeV}$ as the cut-off energy which can be used commonly in the whole density region of interest. This value can be compromised with the constraint $e_{c u t} \gtrsim 50 \mathrm{MeV}$ which we obtained from the condition on the r.m.s. radius of the neutron Cooper pair.

It is interesting to note that cut-off quasiparticle energies around $E_{c u t}=50-70 \mathrm{MeV}$ have been employed in many of recent HFB applications to finite nuclei [9, 10, 11, 25, 28, 61, 85, 86, 87, 88. These cut-off energies are consistent with the constraint $e_{\text {cut }} \sim 50 \mathrm{MeV}$ suggested from the above analysis. Much smaller cut-off energies $\lesssim 10 \mathrm{MeV}$ adopted in early applications of the DDDI [54, 55] are not appropriate from the view point of the spatial structure of the neutron Cooper pair wave function. In Ref. [8] the cut-off energy of $20 \mathrm{MeV}$ for the single-particle energy (40 MeV in the center of mass frame energy) was shown to describe reasonably the scattering wave function at zero energy. This cut-off energy is not very different from the cut-off energy $e_{c u t} \sim 30 \mathrm{MeV}$ which we find most reasonable (among the selected examples) in the lowest density case $\rho / \rho_{0}=1 / 512$. In the the delta interaction model adopted in Ref. [59] the cut-off energy is examined with respect to the low-energy scattering phase shift in the ${ }^{1} S$ channel. The cut-off value adopted is around 5-10 MeV in the single-particle energy $(9-20 \mathrm{MeV}$ for the center of mass frame energy), in disagreement with our value $e_{\text {cut }} \sim 30 \mathrm{MeV}$. The difference seems to originate from different strategies to the delta interaction: the momentum dependence of the interaction matrix element is accounted for by the cut-off energy in Ref.[59] while it is taken into account in the present approach mostly through the density dependent interaction strength $V_{0}[\rho]$.

\section{DDDI parameters}

It is useful to parameterize the interaction strength of the DDDI in terms of a simple function of the density. The following form is often assumed $[8,09,10,11,54,55,58]$ :

$$
\begin{aligned}
V_{0}[\rho] & =v_{0}\left(1-\eta\left(\frac{\rho_{t o t}}{\rho_{c}}\right)^{\alpha}\right), \\
\rho_{c} & =0.16 \mathrm{fm}^{-3}
\end{aligned}
$$

where $\rho_{\text {tot }}$ is the total nucleon density. The parameters $v_{0}, \eta$ and $\alpha$ need to be determined. In the works by Bertsch and Esbensen [8] and Garrido et al. [58] the parameters are determined so that the parameterized DDDI reproduces the pairing gap obtained with the Gogny force in symmetric nuclear matter as well as the experimental s-wave scattering length at zero density. We shall follow a similar line, but we add the important constraint that the spatial structure of the neutron Cooper pair is also reasonably reproduced. As discussed in the subsection just above, this can be achieved if we constrain the cut-off energy to a value around $e_{\text {cut }} \sim 50 \mathrm{MeV}$. Note also our definition of the cut-off energy is different from that in Refs. [8, [58], as mentioned in Subsection $\nabla \mathrm{A}$

Our procedure is as follows. We consider symmetric nuclear matter. The cut-off energy is fixed to $e_{\text {cut }}=50 \mathrm{MeV}$ or $60 \mathrm{MeV}$ for the reasons mentioned above. We then fix the interaction strength $V_{0}[0]=v_{0}$ at zero density to a value 
$v_{0}$ which reproduces the scattering length $a$ in the free space. $v_{0}$ satisfying this condition is given by 8 , 58$]$

$$
\begin{aligned}
& v_{0}=-\frac{2 \pi^{2} \hbar^{2} m^{-1}}{k_{c}-\pi / 2 a}, \\
& k_{c}=\sqrt{2 m e_{c u t}} / \hbar .
\end{aligned}
$$

If we use as the scattering length $a$ in Eq.(17) the one associated with the Gogny force, the pairing gap of the DDDI in the low density limit $\rho / \rho_{0} \rightarrow 0$ coincides with that of the Gogny force. However, since the scattering length $a=-13.5$ fm of the Gogny D1 is slightly off the experimental value, we adopt the experimental one $a=-18.5 \mathrm{fm}$ for Eq.(17). This is equivalent to constrain the DDDI at the low density limit by the bare nucleon force.

To determine the other parameters $\eta$ and $\alpha$ controlling the density-dependence of the interaction strength, we first calculate at several representative points of density the values of $V_{0}$ with which the neutron gap $\Delta_{F}$ of the Gogny D1 force is reproduced. We then search the parameters $\eta$ and $\alpha$ so that the simple function Eq. (15) fits well to the values

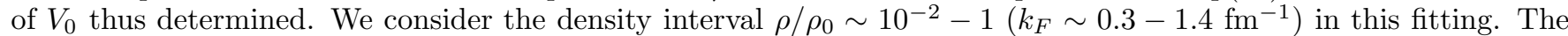
obtained values of the parameters (denoted DDDI-D1) are shown in Table IV where we list also the parameter set obtained when we use $e_{\text {cut }}=60 \mathrm{MeV}$ instead of $50 \mathrm{MeV}$. Another set of the parameters derived in the same way from the Gogny D1S force (DDDI-D1S) is listed. We performed the same procedure also for the G3RS force (DDDI-G3RS).

The pairing gap obtained with these parameterizations of the DDDI are shown in Fig[12 The resultant gap $\Delta$ agrees with that of the corresponding reference gap to the accuracy of about one hundred keV for the whole density region below $\rho / \rho_{0}=1$. Although the results for $e_{c u t}=60 \mathrm{MeV}$ are not shown here, the agreement with the reference gaps is as good as in the $e_{c u t}=50 \mathrm{MeV}$ case.

\begin{tabular}{cccc}
\hline \hline & $v_{0}\left[\mathrm{MeV} \mathrm{fm}^{-3}\right]$ & $\eta$ & $\alpha$ \\
\hline DDDI-D1 & & & \\
$e_{\text {cut }}=50 \mathrm{MeV}$ & -499.9 & 0.627 & 0.55 \\
$e_{\text {cut }}=60 \mathrm{MeV}$ & -458.4 & 0.603 & 0.58 \\
DDDI-D1S & & & \\
$e_{\text {cut }}=50 \mathrm{MeV}$ & -499.9 & 0.652 & 0.56 \\
$e_{\text {cut }}=60 \mathrm{MeV}$ & -458.4 & 0.630 & 0.60 \\
DDDI-G3RS & & & \\
$e_{\text {cut }}=50 \mathrm{MeV}$ & -499.9 & 0.8720 .58 \\
$e_{\text {cut }}=60 \mathrm{MeV}$ & -458.4 & 0.8450 .59 \\
\hline \hline
\end{tabular}

TABLE IV: The parameter sets of the density-dependent delta interaction with the cut-off energies $e_{\text {cut }}=50$ and $60 \mathrm{MeV}$, derived from the procedure applied to the Gogny D1 and D1S, and the G3RS forces. See text for details.

It is noticed in TableIV that the parameter $\alpha$ takes a similar value $\alpha=0.58-0.60$ (in the case of $e_{\text {cut }}=60 \mathrm{MeV}$ ) for all of DDDI-D1, DDDI-D1S, and DDDI-G3RS, while the difference between the Gogny forces (DDDI-D1,-D1S) and the G3RS force (DDDI-G3RS) is readily recognized in the value of $\eta$. It is seen also that the difference in the cut-off energy influences slightly the values of $v_{0}, \eta$ and $\alpha$. If we compare our result with that of Ref. [58], our parameter values $\eta=0.60-0.63$ for the prefactor and $\alpha=0.55-0.58$ for the power imply stronger density dependence in $V_{0}[\rho]$ than that in Ref. [58], where the parameters are determined as $\eta=0.45, \alpha=0.47$ from a similar fitting to the gap with the Gogny D1 force. This is due to the difference in the cut-off schemes mentioned in Subsection $\bar{\nabla}$ Since the chemical potential $\mu$ increases with the density, the energy window measured from the chemical potential $\mu$ decreases with increasing the density in the scheme of Ref. [58] where the cut-off $e(k)<60 \mathrm{MeV}$ is adopted for all density while in our cut-off scheme the energy window is kept constant $e_{c u t}=50,60 \mathrm{MeV}$ independent of the density. Consequently the stronger density dependence in $V_{0}[\rho]$ is needed in our case.

It may be interesting to compare our DDDI parameter sets with those determined phenomenologically from the experimental pairing gap in the ground states of finite nuclei. Such a comparison is made in Fig 13. where the density dependent interaction strength $V_{0}[\rho]$ is plotted. The phenomenological DDDI's employed here are the so called surface and mixed types, for which the prefactor parameter $\eta$ is fixed to $\eta=1$ and $\eta=0.5$, respectively. The power parameter is assumed as $\alpha=1$ for the mixed type[11], while for the surface type we choose here $\alpha=1$ or $1 / 2$. (Note that the power parameter in the surface type DDDI was investigated in Ref. [10], and $1 / 2 \lesssim \alpha \lesssim 1$ is suggested as a reasonable range of the parameter.) The strength of the surface type DDDI used here is $v_{0}=-521 \mathrm{MeV}^{-3}$ for $\alpha=1$, and $v_{0}=-781$ for $\alpha=1 / 2$, taken from Ref. 10$]$, where the value of $v_{0}$ is determined by a Skyrme HFB calculation for ${ }^{120} \mathrm{Sn}$ to reproduce the gap $1.25 \mathrm{MeV}$. The equivalent energy cut-off $60 \mathrm{MeV}$ adopted in the HFB 


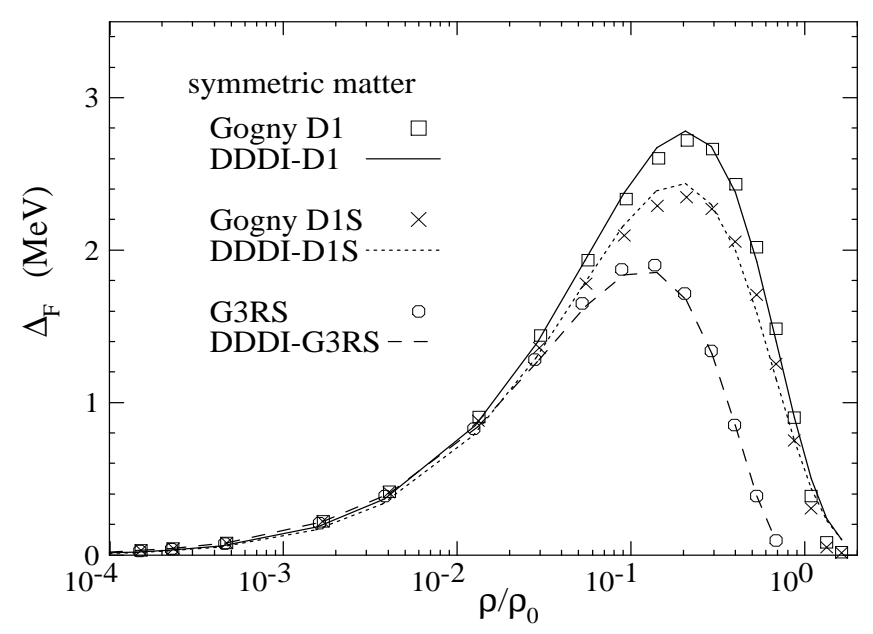

FIG. 12: The pairing gap in symmetric nuclear matter obtained with the DDDI parameter sets shown in Table IV with the cut-off energy $e_{\text {cut }}=50 \mathrm{MeV}$. The solid, dotted and dashed curves represent the result for the parameter sets DDDI-D1, DDDI-D1S, and DDDI-G3RS, respectively. The symbols represent the gap for the reference calculations with the Gogny D1 (square) and D1S (cross) forces, and the G3RS force (circle).

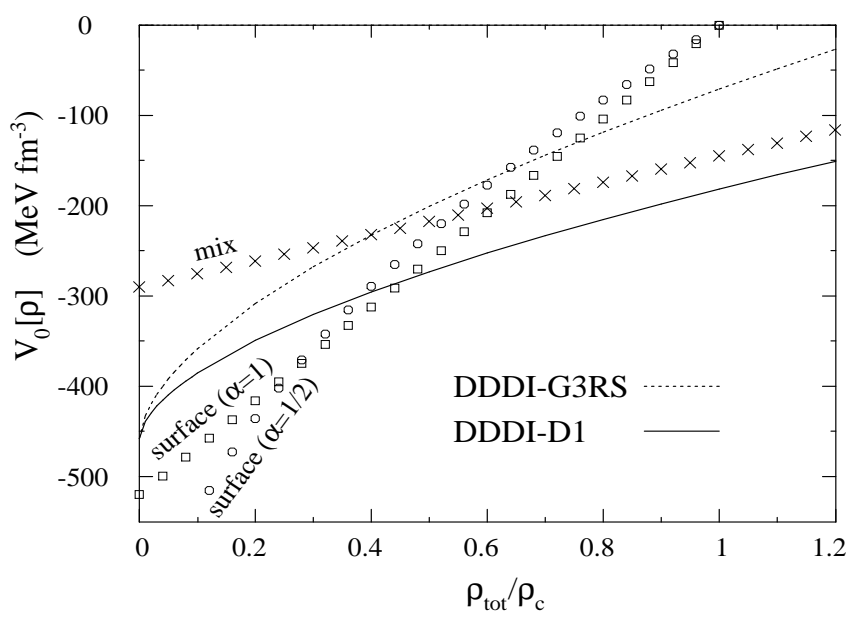

FIG. 13: The density-dependent interaction strength $V_{0}[\rho]$ of the DDDI for the parameter sets DDDI-D1 (solid curve) and DDDI-G3RS (dotted curve) with $e_{\text {cut }}=60 \mathrm{MeV}$. For comparison, $V_{0}[\rho]$ for the phenomenological DDDI parameters of the surface and mixed types are also shown by the symbols. See the text for details.

calculation corresponds to our cut-off $e_{c u t}=60 \mathrm{MeV}$. In the case of the mixed type DDDI, the adopted strength is $v_{0}=-290 \mathrm{MeV} \mathrm{fm}{ }^{-3}$ derived from the same condition on ${ }^{120} \mathrm{Sn}$. It is seen in Fig [13] that the density dependence in the present parameterizations of $V_{0}[\rho]$ is mild in the range $\rho_{t o t} / \rho_{c} \gtrsim 0.4$, resembling more to that of the mixed type DDDI than to that of the surface type. At lower density $\rho_{t o t} / \rho_{c} \lesssim 0.3$ the density dependence is stronger than that of the mixed type DDDI. Note that the interaction strength $V_{0}[\rho]$ itself is not large: it takes the values between those of the mixed and the surface type DDDI's in this low density region. The above comparisons suggest that the density dependence in the present parameterization of $V_{0}[\rho]$ may not be very unrealistic for applications to finite nuclei. It could be also suggested that the present parameterization will be free from the problems pointed to in Ref. [10] for strongly density dependent DDDI's such as the surface type DDDI with small values of the power $\alpha \lesssim 1 / 2$. For more definite conclusions, we need to make more quantitative analyses using the HFB calculation performed directly for finite nuclei. It is also interesting to compare with a new approach to the DDDI with a microscopically derived cut-off factors [89]. This is, however, beyond the scope of this paper, and such analyses will be pursued in future. 


\section{CONCLUSIONS}

We have analyzed the spatial structure of the neutron Cooper pair obtained with the BCS approximation for neutron and symmetric nuclear matter using a bare force and the effective Gogny interaction. The size of the Cooper pair varies significantly with the density: its r.m.s. radius $\xi_{r m s}$ becomes as small as $\sim 5$ fm around $\rho / \rho_{0} \approx 10^{-2}-0.2$, and $\xi_{r m s}$ smaller than the average inter-neutron distance $d$ is realized in a very wide range $\rho / \rho_{0} \approx 10^{-4}-0.1$ at low density. The analysis of the Cooper pair wave function indicates that the probability for the spin up and down neutrons in the pair to be correlated within the average inter-neutron distance $d$ exceeds more than 0.8 in this density range. The strong spatial correlation at short relative distances is also seen for modest density $\rho / \rho_{0} \sim 0.5$, at which the concentration of the pair neutrons within the interaction range $\sim 3 \mathrm{fm}$ reaches about 0.5 . These observations suggest that the spatial di-neutron correlation is strong, at least in the level of the mean-field approximation, in low-density superfluid uniform matter in the wide range of density $\rho / \rho_{0} \approx 10^{-4}-0.5$. The essential feature does not depend on the interactions.

We have investigated the behaviors of the strong di-neutron correlation in connection with the crossover phenomenon between the conventional pairing of the weak coupling BCS type and the Bose-Einstein condensation of the bound neutron pairs. Comparing with the analytic BCS-BEC crossover model assuming a contact interaction, we found that the density region $\rho / \rho_{0} \approx 10^{-4}-10^{-1}$ corresponds to the domain of the BCS-BEC crossover.

We have examined also how the density dependent delta interaction (DDDI) combined with a finite cut-off energy can describe the spatial correlation of the neutron Cooper pair. The spatial correlation at short relative distances and the r.m.s. radius of the pair are described consistently in a wide density region $0<\rho / \rho_{0} \lesssim 1$ provided that we adopt a cut-off energy around $e_{\text {cut }} \sim 50 \mathrm{MeV}$ defined with respect to the chemical potential. We have derived a possible parametrization of the DDDI, which satisfies this new condition on top of the constraints on the gap in symmetric nuclear matter and on the scattering length in the free space. The new DDDI parameterizations may be consistent with or at least not strongly contradictory to the phenomenological DDDI's derived from the gap in finite nuclei.

\section{Acknowledgments}

The author thanks P. Schuck and M. Hjorth-Jensen for useful discussions. He thanks also the Yukawa Institute for Theoretical Physics at Kyoto University and the Institute for Nuclear Theory at University of Washington. Discussions during the YITP workshop YITP-W-05-01 on "New Developments in Nuclear Self-Consistent Mean-Field Theories" and the INT workshop "Towards a universal density functional for nuclei" in the program INT-05-3 "Nuclear Structure Near the Limits of Stability" were useful to complete this work. Discussions with the members of the Japan-U.S. Cooperative Science Program "Mean-Field Approach to Collective Excitations in Unstable Medium-Mass and Heavy Nuclei" are also acknowledged. This work was supported by the Grant-in-Aid for Scientific Research (No. 17540244) from the Japan Society for the Promotion of Science.

[1] A. Bohr and B. R. Mottelson, Nuclear Structure vol. II (Benjamin, 1975).

[2] Y. R. Shimizu, J. D. Garrett, R. A. Broglia, M. Gallardo, and E. Vigezzi, Rev. Mod. Phys. 61, 131 (1989).

[3] T. Takatsuka and R. Tamagaki, Prog. Theor. Phys. Suppl. No. 112, 27 (1993).

[4] U. Lombardo and H.-J. Schulze, Lecture Notes in Physics (Springer 2001), Vol.578, p.30.

[5] D. J. Dean and M. Hjorth-Jensen, Rev. Mod. Phys. 75, 607 (2003).

[6] H. Heiselberg and V. Pandharipande, Ann. Rev. Nucl. Part. Sci, 50, 481 (2000).

[7] D. G. Yakovlev and C. J. Pethick, Ann. Rev. Astron. Astrophys. 42, 169 (2004).

[8] G. F. Bertsch and H. Esbensen, Ann. Phys. 209, 327 (1991).

[9] J. Dobaczewski, W. Nazarewicz, T. R. Werner, J. F. Berger, C. R. Chinn, and J. Dechargé, Phys. Rev. C 53, 2809 (1996).

[10] J. Dobaczewski, W. Nazarewicz, and P.-G. Reinhard, Nucl. Phys. A693, 361 (2001).

[11] J. Dobaczewski and W. Nazarewicz, Prog. Theor. Phys. Suppl. 146, 70 (2002).

J. Dobaczewski, W. Nazarewicz, and M. V. Stoitsov, Euro. Phys. J. A15, 21 (2002).

[12] I. Tanihata, H. Hamagaki, O. Hashimoto, Y. Shida, N. Yoshikawa, K. Sugimoto, O. Yamakawa, T. Kobayashi, N. Takahashi, Phys. Rev. Lett. 55, 2676 (1985).

[13] I. Tanihata, T. Kobayashi, T. Suzuki, K. Yoshida, S. Shimoura, K. Sugimoto, K. Matsuta, T. Minamisono, W. Christe, D. Olson, and H. Wieman, Phys. Lett. B287, 307 (1992).

[14] A. Ozawa, O. Bochkarev, L. Chulkov, D. Cortina, H. Geissel, M. Hellström, M. Ivanov, R. Janik, K. Kimura, T. Kobayashi, A. A. Korsheninnikov, G. Münzenberg, F. Nickel, Y. Ogawa, A. A. Ogloblin, M. Pfützner, V. Pribora, H. Simon, B. Sitár, P. Strmend, K. Sümmerer, T. Suzuki, I. Tanihata, M. Winkler, and K. Yoshida, Nucl. Phys. A691, 599 (2001).

[15] P. G. Hansen and B. Jonson, Europhys. Lett. 4, 409 (1987). Nucl. Phys. A632, 383 (1998). 
[16] K. Ikeda, INS Report JHP-7 (1988); Nucl. Phys. A538, 355c (1992).

17] M. V. Zhukov, B. V. Danilin, D. V. Fedorov, J. M. Bang, I. J. Thompson, and J. S. Vaagen, Phys. Rep. 231, 151 (1993).

[18] F. Barranco, P. F. Bortignon, R. A. Broglia, G. Coló, and E. Vigezzi, Eur. Phys. J. A11, 385 (2001).

[19] S. Aoyama, K. Katō, and K. Ikeda, Prog. Theor. Phys. Suppl. 142, 35 (2001). T. Myo, S. Aoyama, K. Katō, and K. Ikeda, Prog. Theor. Phys. 108, 133 (2002).

[20] K. Hagino and H. Sagawa, Phys. Rev. C 72, 044321 (2005).

[21] D. Sackett, K. Ieki, A. Galonsky, C. A. Bertulani, H. Esbensen, J. J. Kruse, W. G. Lynch, D. J. Morrissey, N. A. Orr, B. M. Sherrill, H. Schulz, A. Sustich, J. A. Winger, F. Deák, Á. Horváth, and Á. Kiss, Z. Seres, J. J. Kolata, R. E. Warner, and D. L. Humphrey, Phys. Rev. C 48, 118 (1993).

[22] S. Shimoura, T. Nakamura, M. Ishihara, N. Inabe, T. Kobayashi, T. Kubo, R. H. Siemssen, I. Tanihata, and Y. Watanabe, Phys. Lett. B348, 29 (1995).

[23] M. Zinser, F. Humbert, T. Nilsson, W. Schwab, H. Simon, T. Aumann, M. J. G. Borge, L. V. Chulkov, J. Cub, Th. W. Elze, H. Emling, H. Geissel, D. Guillemaud-Mueller, P. G. Hansen, R. Holzmann, H. Irnich, B. Jonson, J. V. Kratz, R. Kulessa, Y. Leifels, H. Lenske, A. Magel, A. C. Mueller, G. Münzenberg, F. Nickel, G. Nyman, A. Richter, K. Riisager, C. Scheidenberger, G. Schrieder, K. Stelzer, J. Stroth, A. Surowiec, O. Tengblad, E. Wajda, and E. Zude, Nucl. Phys. A619, 151 (1997).

[24] K. Ieki, A. Galonsky, D. Sackett, J. J. Kruse, W. G. Lynch, D. J. Morrissey, N. A. Orr, B. M. Sherrill, J. A. Winger, F. Deák, Á. Horváth, Á. Kiss, Z. Seres, J. J. Kolata, R. E. Warner, D. L. Humphrey, Phys. Rev. C 54, 1589 (1996).

[25] M. Matsuo, K. Mizuyama, and Y. Serizawa, Phys. Rev. C 71,064326 (2005).

[26] P. Ring and P. Schuck, The Nuclear Many-Body Problem, (Springer-Verlag, 1980).

[27] J. -P. Blaizot and G. Ripka, Quantum Theory of Finite Systems (The MIT press, 1986).

[28] J. Dobaczewski, H. Flocard, and J. Treiner, Nucl. Phys. A422, 103 (1984).

[29] A. Bulgac, preprint FT-194-1980, nucl-th/9907088

[30] A. J. Leggett, in Modern Trends in the Theory of Condensed Matter, Lecture Note in Physics 115, ed. by A. Pekalski and R. Przystawa (Springer-Verlag, Berlin, 1980); J. de Phys. 41, C7-19 (1980).

[31] P. Nozières and S. Schmitt-Rink, J. Low Temp. Phys. 59, 195 (1985).

[32] J. Bardeen, L. N. Cooper, and J. R. Schrieffer, Phys. Rev. 108, 1175 (1957). P. G. de Gennes, Superconductivity of Metals and Alloys (Benjamin 1966). M. Tinkham, Introduction to Superconductivity (McGraw-Hill 1975).

[33] C. A. R. Sá de Melo, M. Randeria, and J. R. Engelbrecht, Phys. Rev. Lett. 71, 3202 (1993).

[34] J. R. Engelbrecht, M. Randeria, and C. A. R. Sá de Melo, Phys. Rev. B 55, 15153 (1997).

[35] M. Randeria, in Bose-Einstein Condensation, ed. by A. Griffin, D. Snoke, and S. Stringari (Cambridge Univ. Press, Cambridge, 1995).

[36] C. A. Regal, M. Greiner, and D. S. Jin, Phys. Rev. Lett. 92, 040403 (2004).

[37] T. Alm, B. L. Friman, G. Röpke, and H. Schulz, Nucl. Phys. A551, 45 (1993).

[38] H. Stein, A. Schnell, T. Alm, and G. Röpke, Z. Phys. A 351, 295 (1995).

[39] M. Baldo, U. Lombardo and P. Schuck, Phys. Rev. C 52, 975 (1995).

[40] U. Lombardo, and P. Schuck, Phys. Rev. C 63, 038201 (2001).

[41] U. Lombardo, P. Nozières, P. Schuck, H.-J. Schulze, and A. Sedrakian, Phys. Rev. C 64, 064314 (2001).

[42] T. Takatsuka, Prog. Theor. Phys. 48, 1517 (1972).

[43] T. Takatsuka, Prog. Theor. Phys. 71, 1432 (1984).

[44] M. Baldo, J. Cugnon, A. Lejeune, and U. Lombardo, Nucl. Phys. A515, 409 (1990).

[45] Ø. Elgarøy, L. Engvik, M. Hjorth-Jensen, E. Osnes, Nucl. Phys. A604, 466 (1996).

[46] V. A. Khodel, V. V. Khodel, and J. W. Clark, Nucl. Phys. 598, 390 (1996).

[47] F. V. De Blasio, M. Hjorth-Jensen, Ø. Elgarøy, L. Engvik, G. Lazzari, M. Baldo, and H.-J. Schulze, Phys. Rev. C 56, 2332 (1997).

[48] M. Serra, A. Rummel, and P. Ring, Phys. Rev. C 65, 014304 (2001).

[49] E. Garrido, P. Sarriguren, E. Moya de Guerra, U. Lombardo, P. Schuck, and H. J. Schulze, Phys. Rev. C 63, 037304 (2001).

[50] H. Kucharek, P. Ring, P. Schuck, R. Bengtsson, and M. Girod, Phys. Lett. B216, 249 (1989).

H. Kucharek, P. Ring, and P. Schuck, Z. Phys. 119, 119 (1989).

[51] A. Sedrakian, T. T. S. Kuo, H. Müther, and P. Schuck, Phys. Lett. B576, 68 (2003).

[52] J. Dechargé and D. Gogny, Phys. Rev. C21, 1568 (1980).

[53] R. R. Chasman, Phys. Rev. C 14, 1935 (1976).

[54] J. Terasaki, P.-H. Heenen, P. Bonche, J. Dobaczewski, and H. Flocard, Nucl. Phys. A593, 1 (1995).

[55] N. Tajima, P. Bonche, H. Flocard, P.-H. Heenen, M. S. Weiss, Nucl. Phys. A551, 434 (1993).

[56] S. A. Fayans and D. Zawischa, Phys. Lett. B383, 19 (1996).

[57] S. A. Fayans, S. V. Tolokonnikov, E. L. Trykov, and D. Zawischa, Nucl. Phys. A676, 49 (2000).

[58] E. Garrido, P. Sarriguren, E. Moya de Guerra, and P. Schuck, Phys. Rev. C 60, 064312 (1999).

[59] H. Esbensen, G. F. Bertsch, and K. Hencken, Phys. Rev. C 56, 3054 (1997).

[60] A. Bulgac and Yongle Yu, Phys. Rev. Lett. 88, 042504 (2002).

A. Bulgac, Phys. Rev. C 65, 051305(R) (2002).

[61] Yongle Yu and A. Bulgac, Phys. Rev. Lett. 90, 222501 (2003). 
[62] M. Matsuo, K. Mizuyama, and Y. Serizawa, J. of Phys. Conf. Ser. 20, 113 (2005); M. Matsuo, Proc. of the YITP workshop "New Developments in Nuclear Self-Consistent Mean-Field Theories", Soryushiron Kenkyu (Kyoto) 112, B59 (2005).

[63] R. Tamagaki, Prog. Theor. Phys. 39, 91 (1968).

[64] G. F. de Téramond and B. Gabioud, Phys. Rev. C 36, 691 (1987).

[65] J. F. Berger, M. Girod, and D. Gogny, Comp. Phys. Comm. 63, 365 (1991).

[66] J. M. C. Chen, J. W. Clark, E. Krotscheck, and R. A. Smith, Nucl. Phys. A451, 509 (1986).

[67] J. M. C. Chen, J. W. Clark, R. D. Davé, and V. V. Khodel, Nucl. Phys. A555, 59 (1993).

[68] T. L. Ainsworth, J. Wambach, and D. Pines, Phys. Lett. B222, 173 (1989).

[69] J. Wambach, T. L. Ainsworth, and D. Pines, Nucl. Phys. A555, 128 (1993).

[70] H.-J. Schulze, J. Cugnon, A. Lejeune, M. Baldo, and U. Lombardo, Phys. Lett. B375, 1 (1996).

[71] H.-J. Schulze, A. Polls, and A. Ramos, Phys. Rev. C 63, 044310 (2001).

[72] U. Lombardo, P. Schuck, and W. Zuo, Phys. Rev. C 64, 021301(R) (2001).

[73] C. Shen, U. Lombardo, P. Schuck, W. Zuo, and N. Sandulescu, Phys. Rev. C 67, 061302(R) (2003).

[74] A. Schwenk, B. Friman, G. E. Brown, Nucl. Phys. A713, 191 (2003).

[75] U. Lombardo, P. Schuck, and C. Shen, Nucl. Phys. A731, 392 (2004).

[76] H. Heiselberg, C. J. Pethick, H. Smith, and L. Viverit, Phys. Rev. Lett. 85, 2418 (2000).

[77] A. Fabrocini, S. Fantoni, A. Y. Illarionov, and K. E. Schmidt, Phys. Rev. Lett. 95, 192501 (2005).

[78] F. Barranco, R. A. Broglia, G. Gori, E. Vigezzi, P. F. Bortignon, and J. Terasaki, Phys. Rev. Lett. 83, 2147 (1999).

[79] J. Terasaki, F. Barranco, R. A. Broglia, E. Vigezzi, and P. F. Bortignon, Nucl. Phys. A697, 127 (2002).

[80] G. Gori, F. Ramponi, F. Barranco, P. F. Bortignon, R. A. Broglia, G. Colò and E. Vigezzi, Phys. Rev. C 72, 011302(R) (2005).

[81] Ø. Elgarøy and M. Hjorth-Jensen, Phys. Rev. C57, 1174 (1998).

[82] M. Marini, F. Pistolesi, G. C. Strinati, Eur. Phys. J. B 1, 151 (1998).

[83] T. Papenbrock, and G. F. Bertsch, Phys. Rev. C 59, 2052 (1999).

[84] H. Heiselberg, Phys. Rev. A 63, 043606 (2001).

[85] M. Grasso, N. Sandulescu, Nguyen Van Giai, and R. J. Liotta, Phys. Rev. C 64, 064321 (2001).

[86] M. V. Stoitsov, J. Dobaczewski, and W. Nazarewicz, S. Pittel, and D. J. Dean, Phys. Rev. C 68, 054312 (2003).

[87] E. Terán, V. E. Oberacker, and A. S. Umar, Phys. Rev. C 67, 064314 (2003).

[88] M. Bender, P.-H. Heenen and P.-G. Reinhard, Rev. Mod. Phys. 75, 121 (2003).

[89] T. Duguet, Phys. Rev. C 69, 054317 (2004). 